\title{
Applications of interval computations to earthquake-resistant engineering: How to compute derivatives of interval functions fast
}

\author{
Vladik Kreinovich, David Nemir, and Efren Gutierrez
}

One of the main sources of destruction during earthquake is resonance. Therefore, the following idea has been proposed. We design special control linkages between floors that are normally unattached to the building but can be attached if necessary. They are so designed that adding them changes the building's characteristic frequency. We continuously monitor displacements within the structure, and when they exceed specified limits, the linkages are engaged in a way to control structural motion. This idea can also be applied to avoid vibrational destruction of large aerospace structures.

\section{Приложение интервальных вычислений к сейсмоустойчивой инженерии: как быстро вычислить производные интервальных функций}

\author{
B. Крейнович, А. Немй, Е. ГУтиэрРеС
}

\begin{abstract}
Один из основных источнихов разрушений при землетрясеняи - резонанс. В связи с этим предложена следуюшая идея. Мы разрабатываем спешиальные управляюшие связи между этажами, которые в норме не соединены с самим зданием, но при необходимости могут быть присоединены. Они созданы так, что нх добавление изменяет резонансную частоту здания Мы последовательно отстеживаем смешения в структуре, и, хогда они превышают опрелеленный прелел, связи вк.лючаются аля хонтроля лвижения системы Эта илея также может быть шрименена, чтобы предотвратить вибрашионное разрушение в больших аэрокосмичесхих системах
\end{abstract}

\section{Brief introduction to an engineering problem}

The problem of resonance destruction. One of the main sources of destruction during earthquake is resonance. The majority of the earthquake strikes are not powerful enough to destroy a building in one blow, but the earthquake usually contains a wide spectrum of vibrations with different frequencies. When the characteristic frequencies of the building lie inside this spectrum area, resonance and eventually destruction can occur.

The vibrations can also occur, when a bridge, an oil platform, or an aerospace construction encounters periodic waves. How can we diminish destruction? Before we enumerate different methods, let us describe (briefly) the corresponding mathematical problem.

(C) V Kreinovich, D Nemir, E. Gutierrez, 1995 
Physical and mathematical description of a resonance: in brief. A building (bridge, aerospace construction, etc) consists of many basic parts (beams, etc). Earthquakes cause these parts to displace, thus (ultimately) destroying the building. The position of each part can be described by describing the positions of its major points (endpoints, sometimes a midpoint, etc). In order to describe the state of a building at any given moment of time $t$, we must describe the positions $\vec{x}_{a}(t), 1 \leq a \leq N$, of all these points. Since we are interested not in the engineering design itself, but only in the abnormalities, instead of the positions themselves, we will use displacements $\Delta \vec{x}_{a}(t)=\vec{x}_{a}(t)-\vec{x}_{a}^{(0)}$, i.e., the differences between the current and ideal positions of these points.

How to describe dynamics? According to Newton's law, to describe the dynamics, we need to know the total force that acts on each point. This force $\vec{F}_{a}$ consists of two parts: $\vec{F}_{a}=\vec{F}_{a}^{e}+\vec{F}_{a}^{s}$ :

- the external force $\vec{F}_{a}^{e}$ that is caused by the earthquake itself;

- the internal, or structural force $\vec{F}_{a}^{s}$ that is caused by the connection between different parts of the building.

The structural force depends on the positions and velocities of all the points. Then, we can get the accelerations $d^{2} \vec{x}_{a}(t) / d t^{2}$ as $\vec{f}_{a}=\vec{F}_{a} / m_{a}$. Thus, we have a system of second-order differential equations:

$$
d^{2} \vec{x}_{a}(t) / d t^{2}=\vec{f}_{a}^{s}\left(\vec{x}_{1}, \ldots, \vec{x}_{N}, \dot{\vec{x}}_{1}, \ldots, \dot{\vec{x}}_{n}\right)+\vec{f}_{a}^{e} .
$$

These equations can be reformulated in terms of displacements:

$$
d^{2} \Delta \vec{x}_{a}(t) / d t^{2}=\vec{f}_{a}^{s}\left(\Delta \vec{x}_{1}, \ldots, \Delta \vec{x}_{N}, \Delta \dot{\vec{x}}_{1}, \ldots, \Delta \dot{\vec{x}}_{n}\right)+\vec{f}_{a}^{e} .
$$

Since we are talking about small and not so fast displacements (large or fast displacements will immediately ruin the building), we can expand $\vec{f}_{a}^{s}$ into Taylor series, and neglect the terms that are quadratic (or of higher order) in $\Delta \vec{x}_{a}$ and $\Delta \dot{\vec{x}}_{\alpha}$. Thus, we arrive at a linear system of second-order differential equations with constant coefficients. It is well how to solve such a system:

- First, we reduce it to a first-order system by introducing new variables $\vec{v}_{a}=\dot{\vec{x}}_{a}$. In terms of $\Delta x_{a}$ and $v_{a}$, we get the following first-order svstem:

$$
\begin{gathered}
\dot{\vec{v}}_{a}=\vec{f}_{a}^{s l}\left(\Delta \vec{x}_{1}, \ldots, \Delta \vec{x}_{n}, \vec{v}_{1}, \ldots, \vec{v}_{n}\right)+\vec{f}_{a}^{e}, 1 \leq a \leq N ; \\
\Delta \vec{x}_{a}=\vec{v}_{a}, 1 \leq a \leq N .
\end{gathered}
$$

Here, the additional index $l$ in the expression for the structural acceleration $\vec{f}_{a}^{s l}$ indicates that we are restricting ourselves to linear terms only.

These equations become easier to grasp and to solve if instead of $2 N$ vector unknowns $\Delta \vec{x}_{a}$ and $\vec{v}_{a}$, we consider all $3 \cdot 2 N=6 N$ components of these vectors as new scalar unknowns $q_{1}, \ldots, q_{6 N}$. In terms of these variables, we get a system of first-order linear differential equations with constant coefficients:

$$
\dot{q}_{a}=\sum A_{a b} q_{b}+f_{a}
$$


- It is well known how to solve such a system (see, e.g., [2]). Namely, if $f_{a}=0$, then a general solution of this system can be represented as linear combination of the functions $\exp \left(\lambda_{\alpha} t\right)$ and $t^{m} \exp \left(\lambda_{\alpha} t\right)$, where $\lambda_{\alpha}$ are eigenvalues of the matrix $A_{a b}$ (terms $t^{m} \exp \left(\lambda_{\alpha} t\right)$ with $m>0$ appear only if we have a degenerate eigenvalue).

These terms have different asymptotic behavior:

- If $\operatorname{Re} \lambda_{\alpha}>0$, then the corresponding term becomes large as $t \rightarrow \infty$.

- If $\operatorname{Re} \lambda_{\alpha}<0$, then the term tends to 0 as $t \rightarrow \infty$.

- If $\operatorname{Re} \lambda_{\alpha}=0$ (i.e., if $\lambda_{\alpha}=i \omega_{\alpha}$ for some real value $\omega_{\alpha}$ ), then we get an infinitely oscillating term $\exp \left(i \omega_{\alpha} t\right)=\cos \left(\omega_{\alpha} t\right)+i \sin \left(\omega_{\alpha} t\right)$. This real value $\omega_{\alpha}$ is called an eigen frequency of the system.

Buildings are usually stable, therefore $\operatorname{Re} \lambda_{\alpha} \leq 0$.

All this was true for $f_{a}=0$. What to do if $f_{a} \neq 0$ ? An arbitrary non-zero force $f_{a}$ can be represented (by means of Fourier transformation) as a linear combination of the sinusuidal waves with different frequencies $\omega$. Since our equations are linear, in order to get the response $q_{a}$ to the initial force, it is sufficient to compute the system's reactions for different frequencies, and then add these reactions.

In particular, if one of the Fourier components of the force $f_{a}$ has a frequency that coincides with one of the eigen frequencies $\omega_{\alpha}$, then the solution behaves as $t \exp \left(i \omega_{\alpha} t\right)$ when $t \rightarrow \infty$. As a result, displacements and/or frequencies become larger and larger, and the building can be destroyed. This phenomenon is called a resomance.

Strictly speaking, for real-life systems, there is always some friction, therefore, usually $\operatorname{Re} \lambda_{\alpha}<0$. The role of this friction is different for small and large frequencies:

- For small frequencies (that correspond to relatively smooth motion), this friction is small and practically negligible (in physical terms: the materials have elastic behavior for such frequencies). Therefore, although for $t \rightarrow \infty$, the displacement will eventually tend to 0 , but meanwhile, for reasonable $t$, its time dependency is indistinguishable from $t \exp \left(i \omega_{\alpha} t\right)$ and thus, the building may be destroyed.

- For bigger frequencies, that correspond to extremely fast changes of displacement and velocity, there is usually a very serious resistance.

To give the reader who is not a specialist in mechanics an idea that this is really the case, let us imaging ourselves pushing a piece of furniture. It is possible (with some force) to move it to and fro slowly, but practically impossible to move to and fro fast.

Because of that, for high eigen frequencies, the resonance displacement will quickly tend to 0 , and therefore, such perturbations are of no serious threat to the building.

So, not all eigen frequencies cause destruction, but only small ones. How to avoid this resonance destruction?

Active control. The brute-force idea is to add a huge auxiliary mass to damp the vibrations. and to forcefully return the structures to their initial positions by applying computer-controlled force (see, e.g., $[5,10,22,31]$; this idea is called actize control). 
In terms of our mathematical model, adding an additional force means adding an additional term to $f_{a}$ that compensates for the unwelcome (resonant) components of $f_{a}$.

This method often works, but it has two main problems:

- this method requires lots of energy for control, and, e.g., spacecraft must be energyefficient;

- if not precisely implemented, this method can pour lots of additional energy into the system in the wrong times, and thus cause additional destruction [17].

Semi-active control. There exist a modification of this method, called semi-active conlrol, where instead of implementing an out-of-phase force, structural changes are made in the system to change its characteristic frequencies and thus avoid the resonance (see, e.g., $[7,10-14]$ ).

A new approach. For semi-active applications, a new method was proposed in [23]. According to this method, we do not add any energy to the system at all. Instead, we design special control links that are normally unattached to the building but can be attached if necessary. They are so designed that adding them changes the building's characteristic frequency. We keep monitoring displacements, and when motion is detected, the control linkages are alternatively engaged and disengaged in such a way that energy in lower (more destructive) modes of vibration is shifted into higher modes, where the energy is quickly dissipated through passive dumping within the structure itself. This idea can be also applied to avoid vibrational destruction of large aerospace structures.

This idea has been thoroughly checked theoretically, and verified through computer simulation [23].

A brief mathematical description of the new approach. In terms of our above-given mathematical description of resonance destruction, the idea is as follows. When we engage an additional linkage, we thus change the way how the displacements and velocities of different points influence each other. In our terms, we change the structural force function $\vec{F}_{a}^{s}$. As a result, the coefficients of the linearized system of equations will also change: instead of

$$
\dot{q}_{a}=\sum A_{a b} q_{b}+f_{a}
$$

we will have

$$
\dot{q}_{a}=\sum \tilde{A}_{a b} q_{b}+f_{a}
$$

$\tilde{A}_{a b} \neq A_{a b}$.

The idea is as follows: if the frequency of one of the components of the external force coincides with one of the eigen frequencies of the system (i.e., with one of the eigen values of the matrix $A_{a b}$ ), then, we engage the control linkage; this changes the matrix and hence, changes the eigenvalues that are no more equal to the frequencies of the external force.

In order to implement this idea, we must have a way to decide when to switch. We have already mentioned that only small frequencies are potentially destructive. Therefore, it is reasonable to estimate the potential danger of the existing displacements and velocities by the total energy of all low-frequency components (i.e., of all the components whose frequency is $\leq \omega_{0}$ for some chosen $\omega_{0}$ ), and to engage the control linkage if and only if this engagement decreases this total energy.

For a linear system, energy is a quadratic function of displacements and velocities (i.e., in our terms, of the variables $q_{\alpha}$ ): terms that are quadratic in $\Delta \vec{x}_{a}$ correspond to the potential 
(elastic) energy, and terms that are quadratic in velocity represent kinetic energy. For this same reason, the total energy of low-frequency components is a quadratic function of $q_{a}$, say $E=\sum E_{a b} q_{a} q_{b}$. When we engage a control linkage, we thus change the coefficients of the linear system, and hence, we change the coefficients in the expression for its energy. The new expression for energy will be $\tilde{E}=\sum \tilde{E}_{a b} q_{a} q_{b}$. The coefficients $E_{a b}$ and $\tilde{E}_{a b}$ can be determined before the control starts, so in course of the actual control, we know them.

So, for given $q_{a}$, the question of whether to engage the linkage or not reduces to checking a simple inequality: $E=\sum E_{a b} q_{a} q_{b}>\tilde{E}=\sum \tilde{E}_{a b} q_{a} q_{b}$, or, what is equivalent, $\sum\left(\tilde{E}_{a b}-E_{a b}\right) q_{a} q_{b}>$ 0 .

In real life, we do not know the exact values of $q_{a}$ (i.e., of displacements and velocities). If we substitute the imprecise values of $q_{a}$ into this inequality, then we may end up with a wrong decision (and in these applications, wrong decisions can be fatal):

- We may not engage the linkage when it is necessary to, and thus fail to avoid destruction, or

- We may engage the linkage when there has been no potential damage to the building, and by engaging this linkage at the wrong time, actually worsen the situation and force destruction.

Since imprecision in $q_{a}$ can lead to grave consequences, it is important to determine $q_{a}$ as accurately as possible.

Related mathematical problem: brief informal description. To check for a resonance, one must know not only the displacements $x\left(t_{i}\right)$, but the rates $\dot{x}\left(t_{i}\right)$ with which they change. The existing velocity sensors are much more expensive than the displacement sensors. So, if we are designing a reasonably cost system, we cannot use velocity sensors. Instead, we must estimate the velocity from the measured (and hence, approximately known) values of the displacements $x(t)$.

We must make control decisions really fast (in milliseconds). Therefore, there is no time to process lots of data. So, when estimating $\dot{x}\left(t_{i}\right)$, we can take into consideration only the measurements in a few consequent points. Hence, all of them belong to a small time interval, and therefore, on this interval, the function $x(t)$ can be well approximated by its first few Taylor expansion terms. In case this interval is sufficiently small, linear approximation is sufficient, so we can assume that a function $x(t)$ is linear. If this is not enough, we must add second order terms, and consider the case when $x(t)$ is quadratic.

A reasonable system must rely on low-cost, reasonably priced sensors. Therefore, the resulting measurements have a non-negligible error. This error leads to an error in the resulting estimate for the derivative. In view of that, it is necessary to design methods of computing derivative that would have the smallest possible error.

What we are going to do. In the present paper, we find the optimal estimates for the derivatives for both cases: when the error is of statistical nature (in which case we know its statistical characteristics), and when this error is systematic (in which case we know only the interval of its possible values).

Interval estimates are considered in Part I, statistical estimates in Part II. Part III is reserved for proofs. 


\section{Part I. Interval estimates}

\section{Interval estimates for the derivative: formulation of a mathematical problem}

\subsection{Definitions}

Definition 1. Suppose that for some integer $n$, we are given $n$ real numbers $t_{1}<\cdots<t_{n}$, $n$ real number $\varepsilon_{i}>0$ and $x_{i}$, and $n$ intervals $F_{i}=\left[x_{i}^{+}, x_{i}^{-}\right], i=1, \ldots, n$, where $x_{i}^{-}=x_{i}-\varepsilon_{i}$ and $x_{i}^{+}=x_{i}+\varepsilon_{i}$. By a function interwal we mean the set $\mathcal{F}$ of all continuous functions $x(t)$ such that for all $i=1, \ldots, n, x\left(t_{i}\right) \in F_{i}$.

Rernark. This definition was, in effect, originally proposed by $\mathrm{R}$. E. Moore (see, e.g., discussions of discrele functions in [20], Section 5.1, and [21], Section 2.5).

Definition 2. Suppose that a function interval $\mathcal{F}$ is given. We say that a class of functions $S$ is comsistent (or compatible) with $\mathcal{F}$ if $S \cap \mathcal{F} \neq \varphi$.

Rennark. In our case, this means that the measurement results (expressed by a function interval $\mathcal{F}$ ) are consistent with the supposition that the unknown function belongs to the class $S$.

Definition 3. Suppose that we are given a function interval $\mathcal{F}$, a class $S$ of differentiable functions that is compatible with $\mathcal{F}$, and a real number $t_{0}$. A real number $d$ is called a possible value of the derivative $d x(t) / d t$ w.r.t. $S$ if $d=\dot{x}\left(t_{0}\right)$ for some $x \in S \cap \mathcal{F}$. The set of all possible values will be called a derivative (or a derivative set) of $\mathcal{F}$ with respect to $S$ in the point $t_{0}$, and denoted by $d \mathcal{F} / d t_{\mid S}\left(t_{0}\right)$.

Remark. In other words, $d$ is a possible value if there exists a function $x(t) \in S$ such that $d x\left(t_{0}\right) / d t=d$ and $\left|x_{i}-x\left(t_{i}\right)\right| \leq \varepsilon_{i}$ for $i=1, \ldots, n$.

Proposition 1. If $S$ is a convex class of functions, then the derivative $d \mathcal{F} / d t_{\mid S}$ is convex, i.e., it is either an interval (open, closed, or semi-open), or a semi-line.

(All the proofs are placed in Part III, for reader's convenience).

Remark. In this paper, we will consider only convex classes of functions, namely, the class of all linear functions, and the class of all quadratic functions. Therefore, the derivative sets will always be intervals (finite or infinite).

Denotations. Let us denote the set of all linear functions $x(t)=a+b t$ by $L$, and the set of all quadratic functions $x(t)=a+b t+c t^{2}$ by $Q$.

\subsection{Main problem: first formulation}

Given $\mathcal{F}$ and $t_{0}$, to check whether $\mathcal{F}$ is compatible with $L$ or $Q$, and to compute the derivatives $d \mathcal{F} / d t_{\mid L}$ and $d \mathcal{F} / d t_{\mid Q}$

It is reasonable first to try $L$, and then, if $L$ is incompatible with $\mathcal{F}$, to try $Q$.

\subsection{How to solve this problem?}

Traditional and interval methods of numerical differentiation are not applicable to this problem. There exist several methods of numerical differentiation, both in traditional numerical mathematics (see, e.g., [4]), and in interval mathematics (see, e.g., Chapter 8 of [1], Section 5.4 of 
$[3],[6,16,18]$, Chapter 11 of [19], [24-28]). However, we cannot directly apply these methods to our problem, because:

- methods of traditional numerical mathematics usually give a numerical estimate, and do not provide us with an interval of possible values; and we have already mentioned that for our applications, this is crucial;

- methods of interval mathernalics are mainly designed for estimating the derivative of the functions that are given by analytical expressions $[1,3,6,18,19,24-26]$; definitions from $[16,27,28]$ are applicable to the case when we have finite interval estimates for $x(t)$ for all $t$, but in our case, we have such estimates only for $t=t_{1}, \ldots, t=t_{n}$.

Comment. In general, there is no way to reconstruct a function for all $t$ from the observations in finitely many points. In our problem, however, as we have already mentioned, high-frequency components of the displacement function go to 0 real fast. As a result, we consider only functions that are formed by low-frequency components and are therefore, very smooth. And if we have a very smooth function that is defined on a small interval, then its linear or quadratic terms provide a practically perfect approximation.

We can apply linear programming. These problems can be solved by reducing them to linear programming. For linear functions $x(t)=a+b t \in L, \dot{x}(t)=b$. Therefore, the upper limit $d^{+}$is the solution of the problem $d \rightarrow \max$ under the condition that $\left|a+b t_{i}-x_{i}\right| \leq \varepsilon_{i}$ for $1 \leq i \leq n$, or, what is equivalent, $-\varepsilon_{i} \leq a+b t_{i}-x_{i} \leq \varepsilon_{i}$. The lower limit $d^{-}$is the solution of the problem $d \rightarrow$ min under the same constraints.

For quadratic functions $x(t)=a+b t+c t^{2}, d=b+2 c t_{0}$, therefore, the limits can be found by solving the following conditional optimization problems: $b+2 c t_{0} \rightarrow \max$ and $b+2 c t_{0} \rightarrow \min$ under the condition that $-\varepsilon_{i} \leq a+b t_{i}+c t_{i}^{2}-x_{i} \leq \varepsilon_{i}, i=1, \ldots, n$.

In both cases, we have linear programming problems, and they can be solved using known polynomial-time linear programming techniques (e.g., Karmarkar's method [9]).

Why is linear. programming not satisfactory. As we have already mentioned, we are in the area of real-time control, therefore, the computation time must be as small as possible. The computational time of an algorithm is roughly proportional to the total number of elementary computational steps (arithmetic operations, comparisons, etc). Karmarkar's method demands $C n^{3.5}$ steps, where $n$ is a number of equations (i.e., in our case, the number of measurements), and $C$ is a rather big constant.

Even if we have several processors working in parallel, the general case of a linear programming program is unlikely to get a dramatic speed-up (more formally, it belongs to the class $P$ that consists, crudely speaking, of problems with worst possible parallelization abilities, see, e.g., [8]).

Why cannot we apply known methods of solving interval linear equations? Our problem can be easily reformulated in interval terms. For example, for a linear case, the problem is to find all possible values of $b$ for which for some $a$, we satisfy the system of interval inclusions: $a+b t_{i}-x_{i} \in\left[-\varepsilon_{i}, \varepsilon_{i}\right], 1 \leq i \leq n$. This system is called an interval linear system (for a general definition, see, e.g., $[29,30]$, and references therein), and the interval of all possible values of $b$ is called an optimal solution of such a system. There exist numerous methods of finding optimal solutions of interval linear systems. These methods have been perfected for many years, and the latest algorithms are very ingenious and fast (for the latest survey, see [30]).

The majority of these methods share the same good property: they are universal in the sense that they are applicable to an arbitrary interval linear system. But this same good property 
is the cause of the common problem of these algorithms, the problem that leads us to the necessity to invent new ones. Namely, the problem of finding an optimal solution to an interval linear system has been proved to be NP-hard [15]. This term means that no matter how smart (and thus fast) an algorithm for solving such system can be, in some cases, its computation time will increase exponentially (i.e., as $a^{n}$ for some $a>1$ ). An exponential function grows so fast that for reasonable $n$, this time quickly exceeds the lifetime of the Universe. The fact that such "worst" cases exist for these algorithms does not invalidate their usage in economic problems, in some optimization problems, etc: if once in a while we do not get the optimal result, or it takes too long to get this result, no big deal.

In our problem (earthquake-resistant engineering) milliseconds do count, and $100 \%$ reliability (i.e., getting results in $100 \%$ of all the cases) is an issue. If once in a while, our system fails, the building (or the spaceship) may be destroyed. Because of that specific feature of our problem, we cannot use the existing universal algorithms. We have therefore to design new ones, that will be applicable to our problems only, but that will have a guaranteed (and small) running time.

\subsection{Final formulation of the problem}

To find algorithms that compute derivative sets faster than the general methods of linear programming.

Comment. If we achieve that, then our algorithm will be faster than $n^{3.5}$ and hence (for sufficiently large $n$ ), its running time will be smaller than an exponential function.

\section{First result: How to compute derivative set if we know that $x(t)$ is linear}

Theorem 1. A function interval $\mathcal{F}$ is compatible with $L$ if and only if $d^{+} \geq d^{-}$, where $d^{+}=\min _{i>j}\left(\left(x_{i}^{+}-x_{j}^{-}\right) /\left(t_{i}-t_{j}\right)\right)$ and $d^{-}=\max _{i>j}\left(\left(x_{i}^{-}-x_{j}^{+}\right) /\left(t_{i}-t_{j}\right)\right)$. If $\mathcal{F}$ and $L$ are compatible, then the derivative of $\mathcal{F}$ with respect to $L$ is equal to $\left[d^{-}, d^{+}\right]$.

Remark. The formulas from Theorem 1 prompt the following natural algorithm for computing $d^{+}$: set an auxiliary element where the current record will be stored to $s:=+\infty$ (in Pascal, to $M a x I n t$ ), and then make two embedded loops for $i$ and for $j$, inside which we update the record values $s$ by assigning $s:=\min \left(s,\left(x_{i}^{+}-x_{j}^{-}\right) /\left(t_{i}-t_{j}\right)\right)$. After the loops are over, $s$ contains the desired value $d^{+}$. A similar natural algorithm can compute $d^{-}$.

Since we are interested in computing the estimates for the derivative as fast as possible, a crucial question is what is the running time of this algorithm. A running time is usually estimated by the total number of elementary computational steps (i.e., comparisons, and arithmetic operations) that we must perform to apply the algorithm. So, let us enumerate the number of computational steps for the above-described algorithm.

Before we proceed, let us make one remark. With one exception, throughout the whole paper, by a computational step, we will mean an arithmetic operation. There will be one exception: while computing the statistical estimate, it is impossible to avoid computing a square root, so, for that case (and for that case only), we will add computing the square root to the list If elementary operations. Let us now return to our algorithm. Since we are given $x_{i}, t_{i}$ and $i_{i}$, we first need to compute $x_{i}^{+}=x_{i}+\varepsilon_{i}$ and $x_{i}^{-}=x_{i}-\varepsilon_{i}$. Each computation takes 1 step, so 
totally, we need $2 n$ steps. After that, computing each pair of expressions $\left(x_{i}^{-}-x_{j}^{+}\right) /\left(t_{i}-t_{j}\right)$ and $\left(x_{i}^{+}-x_{j}^{-}\right) /\left(t_{i}-t_{j}\right)$ takes 5 computational steps (3 subtractions and 2 divisions). Totally, we need to compute $n(n-1) / 2$ such pairs (for all $i<j$ ), so it takes $5 n(n-1) / 2$ computational steps. Now, to compute $d^{+}$, we must compare $n(n-1) / 2$ such expressions, and find the smallest one. We can find the smallest of $n(n-1) / 2$ numbers in $n(n-1) / 2-1$ steps. To compute $d^{-}$, we need the same number of comparisons. So, the total number of computational steps for computing $d^{+}$and $d^{-}$is $2 n+5 n(n-1) / 2+2(n(n-1) / 2-1)=2 n+5 / 2 n^{2}-5 / 2 n+n^{2}-n-2=$ $7 / 2 n^{2}-3 / 2 n-2<7 / 2 n^{2}$. So, we proved the following result:

Theorem 2. For $S=L$, there exist an algorithm that given a function interval $\mathcal{F}$, checks whether it is compatible with $L$, and if it is compatible, computes the derivative of $\mathcal{F}$ in $<3.5 n^{2}$ computational steps.

Remarks.

1) This estimate is much better than for Karmarkar's algorithm.

2) As we have already mentioned, in earthquake-resistant engineering, every millisecond counts. So, although our algorithm is faster than the known ones, it would be nice to make it still faster. Our hope that this can be done is based on the following argument. The algorithm that we have just described is based on the simplest possible idea of solving an interval linear system: namely, wherever in this system we have an interval (in our case, $x_{i} \in\left[x_{i}^{-}, x_{i}^{+}\right]$), we choose one of the possible endpoints. Thus, we get lots of different non-interval linear systems. For each of these systems, we find a solution. By comparing these solutions, we compute the biggest and the smallest values of the unknowns (i.e., compute an optimal solution). This idea is known to be too expensive time-wise: it turns out that not all possible linear systems have to be solved. By cutting down on the number of these systems, we can drastically decrease the running time of the algorithms that find optimal solutions of interval linear systems [29,30]. These "cutting" ideas are not directly applicable to our case (at least we could not figure out how to apply them), but their existence makes us hope that our (rather primitive) algorithm can (probably) be further improved.

3) Another reasonable way to decrease the running time of an algorithm is to run it on a parallel computer (in which several processors can run in parallel, i.e., simultaneously). If we have several processors, then we can indeed decrease the computation time:

Theorem 3. For $S=L$, there exists an algorithm that given a function interval $\mathcal{F}$, checks whether $\mathcal{F}$ is compatible with $L$, and if it is compatible, computes the derivative of $\mathcal{F}$ in parallel. The running time of this algorithm is smaller than the time of $2 \log _{2} n+3$ computational steps.

\section{Second result: How to estimate the derivative in real time}

\subsection{Motivation of the following definitions}

In the previous sections, we considered the situation when we have to apply the algorithm once. However, in the desired applications, we must monitor the derivative, i.e., with every 
new measurement, produce a new estimate. This new estimate must be obtained in real lime, i.e., the computations must be done by the moment of the next measurement. Therefore, the computation that uses $x_{1}, \ldots, x_{n}$, must produce the result before the moment $t_{n+1}$, and can, therefore, spend the computational time $\leq t_{n+1}-t_{n}$.

Usually, the time intervals $t_{i+1}-t_{i}$ are either equal, or approximately equal. In both cases, the smallest $m$ of these differences if positive, and the biggest $M$ is finite: $0<m \leq M<\infty$. Therefore, to estimate the derivatives in the moment $t_{n}$, we must use time $\leq M$. If we denote by $\Delta t$ the time of one elementary computational step, then we conclude that for every $n$, we can use $\leq C$ computational steps, where we denoted $C=M / \Delta t$.

For both algorithms from Section 3 , however, the number of computational steps increases with $n$, so we cannot use them directly for monitoring

For a sequeniul computer, the fact that the computation time increases with $n$ can be easily understood. Indeed, since we must process all $n$ values $x_{1}, \ldots, x_{n}$, to each value we must apply at least one elementary operation. The elementary operations that we considered (arithmetic, comparisons, etc) require at most 2 variables. So, we need at least $n / 2$ elementary operations to process all the values $x_{1}, \ldots, x_{n}$. Therefore, the number of computational steps is $\geq n / 2$, and thus it tends to $\infty$ when $n \rightarrow \infty$.

We cannot use all the values $x_{1}, \ldots, x_{n}$. How many of them can we use?

Since processing $k$ numbers requires $\geq k / 2$ computational steps, and the number of steps that we can use is limited by $M / \Delta t$, we can conclude that $k / 2 \leq M / \Delta t$, i.e., that $k \leq b$, where we denoted $b=2 M / \Delta t$. Therefore, there exists a constant $b$ such that in estimating the derivative, we can process only $b$ values of $x_{i}$.

For a parallel computer, we can have similar estimates. Indeed, the final result of the computation is obtained by using some elementary operation. Each operation can handle only two numbers. Therefore, any computation that can be performed in the time of one computational step, can process at most 2 values $x_{i}$. If we take computations that take the time of 2 computational steps, then we can at best process 2 numbers, each of which is a result of processing at most 2 numbers, i.e., the result can depend on at most 4 different values $x_{i}$. In general, after the time that is necessary to perform $k$ computational steps, we can process at most $2^{k}$ different values $x_{i}$. Therefore, since the time is limited by $\leq M / \Delta t$ computational steps, we can process at most $b=2^{M / \Delta t}$ values.

In both cases, to estimate the derivative, we can process at most $b$ different values of $x_{i}$. So, we must choose $b$ values out of $n$. Since we consider a process that needs monitoring, the value of the derivative can change over time. So, to get the most precise estimate at the moment $t_{n}$, we must consider $b$ latest values $x_{n}, x_{n-1}, \ldots, x_{n+1-b}$ (of course, if $n \leq b$, then we can process all the values $x_{i}$ ).

Let us formulate this situation in mathematical terms.

\subsection{Definitions of real-time algorithms, and the complexity of such algorithms}

Definition 4. Suppose that an integer $b>1$ is given. Suppose also that $t_{1}<t_{2}<\cdots<$ $t_{n}<\cdots, x_{1}, x_{2}, \ldots, x_{n}, \ldots$, and $\varepsilon_{1}>0, \varepsilon_{2}>0, \ldots, \varepsilon_{n}>0, \ldots$ are three potentially infinite sequences of real numbers. The values $t_{i}$ will be called moments of time. For a moment $t_{n}$, by a b-bonended function interval $\mathcal{F}_{b}$ we mean the set of all continuous functions $x(t)$ such that $x\left(t_{i}\right) \in F_{i}=\left[x_{i}-\varepsilon_{i}, x_{i}+\varepsilon_{i}\right]$ for all $i$ such that $n-b<i \leq n$. By a b-bounded interial estimate for 
APPLICATIONS OF INTERVAL COMPUTATIONS TO EARTHQUAKE-RESISTANT ENGINEERING. .

a derivative in the moment $t_{n}$, we mean the derivative set $d \mathcal{F}_{\mid b} / d t_{\mid S}$ of the $b$-bounded function interval.

Remark. In particular, for $S=L$, the b-bounded interval estimate for a derivative is equal to $\left[d_{n}^{-}, d_{n}^{+}\right]$, where

$$
d_{n}^{+}=\min _{n \rightarrow b<j<i \leq n} \frac{x_{i}^{+}-x_{j}^{-}}{t_{i}-t_{j}}
$$

and

$$
d_{n}^{-}=\max _{n-b<j<i \leq n} \frac{x_{i}^{-}-x_{j}^{+}}{t_{i}-t_{j}} .
$$

Definition 5. Suppose that an integer $b>1$ and a positive real number $C$ are given. We say that an algorithm computes interval estimates in real time wilh $\leq C$ compulational steps per measurement if this algorithm works as follows: it reads $x_{1}, t_{1}, x_{2}, t_{2}$, then after $\leq C$ computational steps generates $d_{2}^{-}$and $d_{3}^{+}$; then reads $x_{3}$ and $t_{3}$, and in $\leq C$ computational steps generates $d_{3}^{-}$and $d_{3}^{+}$, etc.

Rernark. In principle, one can apply an algorithm from Theorem 2 to estimate $d_{n}^{-}$and $d_{n}^{+}$for all $n$. Since we are processing $\leq b$ numbers, this algorithm will require $C \approx 3.5 b^{2}$ computational steps per measurement. The following theorem shows that we can do better.

Theorem 4. For $S=L$, there exists an algorithm that computes interval estimates in real time, with $<b^{2}+4 b$ computational steps per measurement.

Remark. For parallel computers, if we apply an algorithm from Theorem 3 on every step, we get the following result:

Theorem 5. For $S=L$, there exists an algorithm that computes interval estimates in parallel in real time, with $<2 \log _{2} b+3$ computational steps per measurement.

\section{Third result: How to compute derivative set if $x(t)$ is quadratic}

Theorem 6. Assume that $t_{0}$ is a real number.

If $n \leq 2$, then any function interval $\mathcal{F}$ is compatible with $Q$, and the derivative of $\mathcal{F}$ with respect to $Q$ in the point $t_{0}$ coincides with $(-\infty,+\infty)$.

If $n>2$, then a function interval $\mathcal{F}$ is compatible with $Q$ if and only if the following three conditions are satisfied:

1) $\left(x_{i}^{-}-x_{j}^{+}\right) /\left(t_{i}-t_{j}\right) \leq\left(x_{k}^{+}-x_{l}^{-}\right) /\left(t_{k}-t_{l}\right)$ for all $i, j, k, l$ such that $\left(t_{i}-t_{0}\right)^{2}>\left(t_{j}-t_{0}\right)^{2}$, $\left(t_{k}-t_{0}\right)^{2}>\left(t_{l}-t_{0}\right)^{2}$, and $t_{i}+t_{j}-2 t_{0}=t_{k}+t_{l}-2 t_{0}>0$

2) $\left(x_{j}^{+}-x_{i}^{-}\right) /\left(t_{j}-t_{i}\right) \geq\left(x_{l}^{-}-x_{k}^{+}\right) /\left(t_{l}-t_{k}\right)$ for all $i, j, k, l$ such that $\left(t_{i}-t_{0}\right)^{2}>\left(t_{j}-t_{0}\right)^{2}$, $\left(t_{k}-t_{0}\right)^{2}>\left(t_{l}-t_{0}\right)^{2}$, and $t_{i}+t_{j}-2 t_{0}=t_{k}+t_{l}-2 t_{0}<0$;

3) $d^{+} \geq d^{-}$, where $d^{+}=\min \left(d_{1}^{+}, d_{2}^{+}\right), d^{-}=\max \left(d_{1}^{-}, d_{2}^{-}\right)$,

$d_{2}^{-}=\max \left(N_{i j k l}^{-} / D_{i j k l}\right), d_{2}^{+}=\min \left(N_{i j k l}^{+} / D_{i j k l}\right)$,

$$
N_{i j k l}^{-}=\frac{x_{i}^{-}-x_{j}^{+}}{\left(t_{i}-t_{j}\right)\left(t_{i}+t_{j}-2 t_{0}\right)}-\frac{x_{k}^{+}-x_{l}^{-}}{\left(t_{k}-t_{l}\right)\left(t_{k}+t_{l}-2 t_{0}\right)},
$$




$$
\begin{gathered}
N_{i j k l}^{+}=\frac{x_{i}^{+}-x_{j}^{-}}{\left(t_{i}-t_{j}\right)\left(t_{i}+t_{j}-2 t_{0}\right)}-\frac{x_{k}^{-}-x_{l}^{+}}{\left(t_{k}-t_{l}\right)\left(t_{k}+t_{l}-2 t_{0}\right)}, \\
D_{i j k l}=\frac{1}{t_{k}+t_{l}-2 t_{0}}-\frac{1}{t_{i}+t_{j}-2 t_{0}}
\end{gathered}
$$

min and $\max$ are taken over all quadruples $(i, j, k, l)$ such that $\left(t_{i}-t_{0}\right)^{2}>\left(t_{j}-t_{0}\right)^{2}$, $\left(t_{k}-t_{0}\right)^{2}>\left(t_{l}-t_{0}\right)^{2}$, and $t_{i}+t_{j}-2 t_{0}>t_{k}+t_{l}-2 t_{0}$,

and $d_{1}^{-}=\max \left(\left(x_{i}^{-}-x_{j}^{+}\right) /\left(t_{i}-t_{j}\right)\right), d_{1}^{+}=\min \left(\left(x_{i}^{+}-x_{j}^{-}\right) /\left(t_{i}-t_{j}\right)\right)$, where min and max are taken over all $i>j$ such that $\left(t_{i}-t_{0}\right)^{2}=\left(t_{j}-t_{0}\right)^{2}$.

If $\mathcal{F}$ and $Q$ are compatible, then the derivative of $\mathcal{F}$ with respect to $Q$ in the point $t_{0}$ is equal to $\left[d^{-}, d^{+}\right]$.

Theorem 7. For $S=Q$, there exist an algorithm that given a function interval $\mathcal{F}$ and a point $t_{0}$, checks whether $\mathcal{F}$ is compatible with $Q$, and, if it is compatible, computes the derivative set. This algorithm requires $<n^{4}+12 n^{2}$ computational steps.

Remark. For big $n$, this method is worse than Karmarkar's (that gives $C n^{3.5}$ ). However, for small $n$, it is reasonable to use, because a constant $C$ in Karmarkar's method is rather big.

Theorem 8. For $S=Q$, there exists an algorithm that given a function interval and a value $t_{0}$, checks whether $\mathcal{F}$ is compatible with $Q$, and, if it is compatible, computes the derivative of $\mathcal{F}$ in parallel. The running time of this algorithm is less than or equal to the time necessary for $4 \log _{2} n+5$ computational steps.

We can apply these algorithms to the case of real-time estimates, and get the following results:

Theorem 9. For $S=Q$, there exists an algorithm that computes interval estimates in real time with $<b^{4}+12 b^{2}$ computational steps per measurement.

Theorem 10. For $S=Q$, there exists an algorithm that computes interval estimates in real time in parallel with $\leq 4 \log _{2} b+5$ computational steps per measurement.

\section{Part II. Statistical estimates}

\section{Statistical estimates of the derivative: definitions and the main result}

Remark. In this section, we will consider the case when all the measurements are performed by the same measuring device, and therefore, all the measurements have the same precision.

Denotation. For a random variable $\xi$, we will denote its mathematical expectation (average) by $E[\xi]$, and its standard deviation $\sqrt{E\left[(\xi-E[\xi])^{2}\right]}$ by $\sigma[\xi]$.

Definition 6. Assume that we are given an integer $n \geq 2$, a real number $\sigma>0$, and $n$ real numbers $t_{1}<t_{2}<\cdots<t_{n}$. By a statistical estimate for an interval derivative we mean a linear function that transforms a sequence $x_{1}, \ldots, x_{n}$ into a value $d=a_{1} x_{1}+a_{2} x_{2}+\cdots+a_{n} x_{n}$. We say that a statistical estimate is non-biased if for arbitrary real numbers $a$ and $b$, and for arbitrary $n$ independent random variables $\xi_{i}$ with average 0 and standard deviation $\sigma$, the mathematical expectation $E[d]=E\left[a_{1} x_{1}+a_{2} x_{2}+\cdots+a_{n} x_{n}\right]$ is equal to $b$, where $x_{i}=a+b t_{i}+\xi_{i}$. By an error of a statistical estimate we mean the standard deviation $\sigma[b-d]$ of the difference between 
$b$ and $d$. We say that a statistical estimate is optimal if it is non-biased, and its error is the smallest possible (among non-biased estimates).

Remark. We are interested in statistical estimates for which the error is the smallest possible. We consider only linear functions as statistical estimates, because for the most common distribution (Gaussian) it is known that the estimates for which standard deviation is minimal are linear.

Theorem 11. The optimal statistical estimate is $d=\sum_{i} a_{i} x_{i}$, where

$$
a_{i}=\left(t_{i}-\bar{t}\right) /\left(n \sigma_{t}^{2}\right), \bar{t}=\sum_{i} t_{i} / n, \text { and } \sigma_{t}^{2}=\left(\sum_{i}\left(t_{i}-\bar{t}\right)^{2}\right) / n \text {. }
$$

Its error is equal to $\sigma /\left(\sqrt{n} \sigma_{t}\right)$.

\section{Computational complexity of the optimal statistical estimate}

Case when the times $t_{i}$ are known beforehand. Let us first consider the case, when the times $t_{i}$ are known before the measurements, so that the coefficients $a_{i}$ can be precomputed.

Theorem 12. If the coefficients $a_{i}$ are known, then to compute the optimal statistical estimate for the derivative, we need $2 n-1$ computational steps.

Theorem 13. If the coefficients $a_{i}$ are known, then we can compute the optimal statistical estimate for the derivative in parallel in the time that is necessary for $\leq \log _{2} n+2$ computational steps.

General case. Let us now consider the general case, when the values of $t_{i}$ are not known beforehand.

Theorem 14. In the general case, to compute the optimal statistical estimate for the derivative, we need $\leq 7 n-2$ computational steps.

Theorem 15. If the general case, we can compute the optimal statistical estimate for the derivative in parallel in the time that is necessary for $\leq 3 \log _{2} n+8$ computational steps.

\section{Statistical estimates in real time}

Definition 7. Suppose that an integer $b$ is given. Suppose also that $x_{1}, x_{2}, \ldots, x_{n}, \ldots$ and $t_{1}<t_{2}<\cdots<t_{n}<\cdots$ are two potentially infinite sequence of real numbers. By a stitistical estimate for the derivative in the moment $t_{n}, n \geq b$, we mean the result of applying an optimal statistical estimate for the derivative to the values $x_{n}, x_{n-1}, \ldots, x_{n+1-b}$ and $t_{n}, t_{n-1}, \ldots, t_{n+1-b}$. Definition 8. Suppose that an integer $b>1$ and a positive real number $C$ are given. We say that an algorithm computes statistical estimates for the derivative in real time, with $\leq C$ computational steps per measurement if this algorithm works as follows: it reads $x_{1}, t_{1}$, then after $\leq C$ computational steps reads $x_{2}$ and $t_{2}, \ldots$, reads $x_{b}, t_{b}$, after $\leq C$ computational steps computes a statistical estimate for the derivative in the moment $t_{b}$ and its error, then reads $x_{b+1}, t_{b+1}$, after $\leq C$ computational steps computes a statistical estimate for the derivative in the moment $t_{b+1}$ and its error, etc.

Rernark. Since we are interested also in computing the error, and the formula for the error contains a square root, we must add square root to the list of elementary computational steps. The results are as follows: 
Theorem 16. There exists an algorithm that computes statistical estimates for the derivative in real time, with $\leq 18$ computational steps per measurement.

Remark. The nice property of this algorithm is that its running time does not depend on $b$, so, we can use arbitrarily big $b$, and still get the estimates in real time. This running time can be further diminished if we have several processors at our disposal:

Theorem 17. There exists an algorithm that computes statistical estimates for the derivative in parallel in real time, with $\leq 7$ computational steps per measurement.

Remark. In the next Section, we will consider a frequent case, when $t_{i+1}-t_{i}=$ const. In this case, as we will see, the number of computational steps can be made even smaller.

\section{Optimal statistical estimates for the case when measurements are made in consequent moments of time}

Definition 9. We say that the measurements are made in consequent moments of time if $t_{i+1}-t_{i}=\Delta t$ for some $\Delta t>0$. In this case, $t_{i}=t_{1}+(i-1) \Delta t$.

For this case, we can simplify the formulas from Theorem 11. Let us first consider the case, when $n$ is odd: $n=2 k+1$. To simplify the formulas, let us denote the midpoint $\left(t_{k+1}\right)$ by $s_{0}$, and other points by $s_{-k}, \ldots, s_{-1}, s_{0}, \ldots, s_{1}, \ldots, s_{k}$, where $s_{i}=t_{i+k+1}$. Correspondingly, let us denote by $y_{i},-k \leq i \leq k$, the values that correspond to the points $s_{i}$, i.e., $y_{i}=x_{i+k+1}$.

Proposition 2. If $n=2 k+1$, then the optimal statistical estimate is

$$
d=\sum_{i=-k}^{k} \frac{3 i y_{i}}{k(k+1)(2 k+1) \Delta t}
$$

and its error is equal to $\sigma /(\delta t \sqrt{n k(k+1) / 3})$.

In particular, for $n=3$, the optimal statistical estimate is

$$
d=\frac{y_{1}-y_{-1}}{2 \Delta t}
$$

its error is equal to $\sigma /(\sqrt{2} \Delta t)$; for $n=5$,

$$
d=\frac{2 y_{2}+y_{1}-y_{-1}-2 y_{-2}}{10 \Delta t}
$$

and $\sigma /(\sqrt{2} \Delta t)$; etc.

Let us now consider the case, when $n$ is even: $n=2 k$ for some $k$. In this case, we will also denote the midpoint by $s_{0}: s_{0}=\left(t_{1}+t_{n}\right) / 2=t_{1}+(n / 2-1 / 2) \Delta t$. In this case, the distance between $s_{0}$ and $t_{i}$ is not proportional to $\Delta t$, so it sounds reasonable to introduce the following denotations: we will denote $t_{i}$ by $s_{j}$, where $j=\left(t_{i}-s_{0}\right) / \Delta t=i-(n+1) / 2$, and correspondingly $x_{i}$ by $y_{j}$ for the same $j$. Then $j$ runs from $-(n / 2-1 / 2)$ to $+(n / 2-1 / 2)$. Proposition 3. If $n=2 k$, then the optimal statistical estimate is

$$
d=\sum_{i=-(k-1 / 2)}^{k-1 / 2} \frac{12 i y_{i}}{n\left(n^{2}-1\right) \Delta t}
$$


and its error is equal to $\sigma /\left(\Delta t \sqrt{n\left(n^{2}-1\right) / 12}\right)$.

In particular, for $n=2$, the optimal statistical estimate is

$$
d=\frac{y_{1 / 2}-y_{-1 / 2}}{\Delta t}
$$

and error is equal to $\sigma / \Delta t$; for $n=4$, the optimal statistical estimate is

$$
d=\frac{(3 / 2) y_{3 / 2}+(1 / 2) y_{1 / 2}-(1 / 2) y_{-1 / 2}-(3 / 2) y_{-3 / 2}}{5 \Delta t}
$$

and error is equal to $\sigma /(\sqrt{5} \Delta t)$; etc.

Let us now consider real-time algorithms. The values of $t_{i}$ are known beforehand and therefore, definitions from Section 7 can be simplified:

Definition 10. Suppose that an integer $b$ and real numbers $t_{1}$ and $\Delta t>0$ are given. Suppose also that $x_{1}, x_{2}, \ldots, x_{n}, \ldots$ is a potentially infinite sequence of real numbers. $B y$ a statistical estimate for the derivative in the moment $t_{n}, n \geq b$, we mean the result of applying an optimal statistical estimate for the derivative to the values $x_{n}, x_{n-1}, \ldots, x_{n+1-b}$ and $t_{n}, t_{n-1}, \ldots, t_{n+1-b}$, where $t_{i}=t_{1}+(i-1) \Delta t$.

Definition 11. Suppose that an integer $b>1$ and a positive real number $C$ are given. We say that an algorithm computes statistical estimates for the derivative in real time, with $\leq C$ computational steps per measurement if this algorithm works as follows: it reads $x_{1}$, then after $\leq C$ computational steps reads $x_{2}, \ldots$, reads $x_{b}$, after $\leq C$ computational steps computes a statistical estimate for the derivative in the moment $t_{b}$, then reads $x_{b+1}$, after $\leq C$ computational steps computes a statistical estimate for the derivative in the moment $t_{b+1}$, etc.

Remark: In case $t_{i+1}-t_{i}=\Delta t=$ const, the error does not depend on $i$ or $x_{i}$, so it can be computed before any measurements are known. Therefore, we do not have to consider computations of the error if we are talking about real time algorithms.

Theorem 18. There exists an algorithm that computes statistical estimates for the derivative in real time, with $\leq 10$ computational steps per measurement.

Remark. According to Propositions 2 and 3, we can precompute the coefficients $a_{i}$ of the optimal statistical estimate, and thus computing this estimate would take at most $2 b-1$ computational steps: $b$ multiplications and $b-1$ additions. Therefore, if $b \leq 5$, and $2 b-1<10$, it is better to use the direct formulas described after Propositions 2 and 3 . If $b>6$, then $2 b-1>10$, and it is better to use the algorithm from Theorem 18.

Theorem 19. There exists an algorithm that computes statistical estimates for the derivative in parallel in real time, with $\leq 5$ computational steps per measurement.

Remark. The gain in running time is even bigger than we can conclude from comparing these Theorems from the ones from the previous section. The reason is that in Section 7 we counted computation of a square root as 1 computational step, while in the present Section, we need only arithmetic operations, and arithmetic operations are much faster than computing square root. So, not only we need fewer computational steps, but the steps are shorter.

\section{Optimal statistical estimates in case the function $x(t)$ is quadratic}

Definition 12. Assume that we are given an integer $n \geq 2$, a real number $\sigma>0$, and $n+1$ real numbers $t_{0}$ and $t_{1}<t_{2}<\cdots<t_{n}$. By a linear estimate for an interval derivative at 
the point $t_{0}$, we mean a linear function that transforms a sequence $x_{1}, \ldots x_{n}$ into a value $d=a_{1} x_{1}+a_{2} x_{2}+\cdots+a_{n} x_{n}$. We say that a statistical estimate is non-butsed if for arbitrary real numbers $a, b$ and $c$, and for arbitrary $n$ independent random variables $\xi_{i}$ with average 0 and standard deviation $\sigma$, the mathematical expectation $E[d]=E\left[a_{1} x_{1}+a_{2} x_{2}+\cdots+a_{n} x_{n}\right]$ is equal to $b$, where $x_{i}=a+b\left(t_{i}-t_{0}\right)+c\left(t_{i}-t_{0}\right)^{2}+\xi_{i}$. By an error of a statistical estimate we mean the standard deviation $\sigma[b-d]$ of the difference between $b$ and $d$. We say that a statistical estimate is optimal if it is non-biased, and its error is the smallest possible (among non-biased estimates).

Theorem 20. If $x(t)$ can be quadratic, then the optimal statistical estimate is equal to $d=\sum_{i} a_{i} x_{i}$, where $a_{i}=\alpha+\beta\left(t_{i}-t_{0}\right)+\gamma\left(t_{i}-t_{0}\right)^{2}$, where $\alpha, \beta$ and $\gamma$ are the solutions of the following system of linear equations:

$$
\begin{array}{ll}
\alpha \sum_{i} 1 & +\beta \sum_{i}\left(t_{i}-t_{0}\right)+\gamma \sum_{i}\left(t_{i}-t_{0}\right)^{2}=0 \\
\alpha \sum_{i}\left(t_{i}-t_{0}\right)+\beta \sum_{i}\left(t_{i}-t_{0}\right)^{2}+\gamma \sum_{i}\left(t_{i}-t_{0}\right)^{3}=1 \\
\alpha \sum_{i}\left(t_{i}-t_{0}\right)^{2}+\beta \sum_{i}\left(t_{i}-t_{0}\right)^{3}+\gamma \sum_{i}\left(t_{i}-t_{0}\right)^{4}=0
\end{array}
$$

Remark. In this case, we can repeat Definitions 7 and 8 to define real-time algorithms and their complexity. The result of this application is as follows:

Theorem $21(x(t)$ is quadratic). There exists a constant $C>0$ such that for every $b$, there exists an algorithm that computes statistical estimates for the derivative in real time, with $\leq C$ computational steps per measurement.

Remark. In other words, this algorithm has the same nice property as an algorithm from Theorem 16: its running time does not depend on $b$, so, we can use arbitrarily big $b$, and still get the estimates in real time.

\section{Linear formulas are not helpful for interval estimates of derivatives}

Linear formulas are very simple to compute, so let's try them. In Section 6, we considered formulas that describe an estimate for the derivative as a linear combination of $x_{i}: d=\sum_{i} a_{i} x_{i}$. Computing a linear formula is computationally very easy, so it is reasonable to ask: can we use linear estimates in the interval case as well? We will prove that even in the simplest case, when the function is linear (i.e., $S=L)$, and the precision is fixed $\left(\varepsilon_{i}=\varepsilon\right)$, linear formulas are not helpful for interval estimates.

Definition 13. Assume that we are given an integer $n \geq 2$, a real number $\varepsilon>0$, and $n$ real numbers $t_{1}<t_{2}<\cdots<t_{n}$. By a linear estimate for an interval derivative we mean a linear function that transforms a sequence $x_{1}, \ldots, x_{n}$ into a value $d=a_{1} x_{1}+a_{2} x_{2}+\cdots+a_{n} x_{n}$. By a precision of a linear estimate we mean the maximum possible value of the difference $|b-d|$ for all $a, b$ and $x_{i}$ such that $\left|x_{i}-\left(a+b t_{i}\right)\right| \leq \varepsilon$.

Remark. So, if the precision is 0.1 , it means that the real values $b$ of the derivative and the estimate $d$ can differ by $\leq 0.1$, and therefore, the possible values of the derivative belong to the interval $[d-0.1, d+0.1]$. Naturally, we are interested in the estimates with the best (i.e., the smallest) value of the precision.

Theorem 22. For given $n, t_{1}, \ldots, t_{n}$, a linear estimate has the smallest possible precision when $a_{n}=-a_{1}=1 /\left(t_{n}-t_{1}\right)$, and $a_{2}=a_{3}=\cdots=a_{n-1}=0$. 
Remark. So, the best estimates for the linear formulas are obtained when we use only 2 measurements, and the resulting estimate is the simplest numerical differentiation $\left(x_{n}-x_{1}\right) /\left(t_{n}-\right.$ $t_{1}$ ). In other words, if we restrict ourselves to linear estimates, then there is no way to use additional measurements to improve the precision of our estimates of the derivative. This means that in case the error of the simple numerical estimate is too big, linear formulas cannot help in diminishing this error. In other words, linear formulas are not helpful for interval estimates of derizuatives.

\section{Part III. Proofs}

Proof of Proposition 1. Suppose that $S$ is convex, and $d_{1}$ and $d_{2}$ are possible values of the derivative. This means that $d_{1}=\dot{x}_{1}\left(t_{0}\right)$ and $d_{2}=\dot{x}_{2}\left(t_{0}\right)$ for some functions $x_{i} \in S \cap \mathcal{F}$. Let us prove that for every $\alpha \in[0,1], \alpha d_{1}+(1-\alpha) d_{2}$ is also a possible value. Indeed, since $S$ is convex, and (as is easy to prove) $\mathcal{F}$ is convex, the function $x(t)=\alpha x_{1}(t)+(1-\alpha) x_{2}(t)$ also belongs to $S \cap \mathcal{F}$, and therefore its derivative $d x\left(t_{0}\right) / d t=\alpha d x_{1}\left(t_{0}\right) / d t+(1-\alpha) d x_{2}\left(t_{0}\right) / d t=\alpha d_{1}+(1-\alpha) d_{2}$ is a possible value.

Proof of Theorem 1. A linear function $x(t)=a+b t$ belongs to a function interval $\mathcal{F}$ if and only if $x_{i}^{-} \leq a+b t_{i} \leq x_{i}^{+}$for all $i$ from 1 to $n$. We are interested in the values of $d x(t+0) / d t=b$, so let us eliminate $a$ from these inequalities. To do that, suppose that $b$ is given, and let us find whether there exists an $a$ for which the function $x(t)=a+b t$ satisfies all these inequalities. If we move $b t_{i}$ into the other side of the inequalities, we get the following equivalent inequalities: $x_{i}^{-}-b t_{i} \leq a \leq x_{i}^{+}-b t_{i}$. So, $a$ must be not bigger than all the numbers $x_{i}^{+}-b t_{i}$, and not smaller than all the numbers $x_{i}^{-}-b t_{i}$. In other words, $a$ must lie between $\max _{j}\left(x_{j}^{-}-b t_{j}\right)$ and $\min _{i}\left(x_{i}^{+}-b t_{i}\right)$. Such a number exists if and only if $\min _{i}\left(x_{i}^{+}-b t_{i}\right) \geq \max _{j}\left(x_{j}^{-}-b t_{j}\right)$, i.e., if and only if $x_{i}^{+}-b t_{i} \geq x_{j}^{-}-b t_{j}$ for every $i$ and $j$.

So, the problem is reduced to the following: find the interval of all values of $b$ for which $x_{i}^{+}-b t_{i} \geq x_{j}^{-}-b t_{j}$ for every $i$ and $j$. By moving all terms with $b$ to the right-hand side, we can transform this inequality into the following: $b\left(t_{i}-t_{j}\right) \leq x_{i}^{+}-x_{j}^{-}$. This inequality must be true for every pair $(i, j)$. If $i=j$, it degenerates into $0 \leq 0$, and is thus trivially true. If $i>j$, then $t_{i}>t_{j}$, and this inequality turns into $b \leq\left(x_{i}^{+}-x_{j}^{-}\right) /\left(t_{i}-t_{j}\right)$. When $i<j$, then $t_{i}<t_{j}$, and the above inequality turns into $b \geq\left(x_{j}^{-}-x_{i}^{+}\right) /\left(t_{j}-t_{i}\right)$. So, $b$ must be not smaller than all the numbers $\left(x_{j}^{-}-x_{i}^{+}\right) /\left(t_{j}-t_{i}\right)$, and not greater than all the numbers $\left(x_{i}^{+}-x_{j}^{-}\right) /\left(t_{i}-t_{j}\right)$. These inequalities are equivalent to the condition that $b$ is not smaller than the biggest of its lower bounds $\max _{j>i}\left(\left(x_{j}^{-}-x_{i}^{+}\right) /\left(t_{j}-t_{i}\right)\right)$, and not bigger than the smallest of its upper bounds $\min _{i>j}\left(\left(x_{i}^{+}-x_{j}^{-}\right) /\left(t_{i}-t_{j}\right)\right)$.

In other words, $b$ is a possible value of the derivative if and only if $d^{-} \leq b \leq d^{+}$. Such values exist if and only if $d^{-} \leq d^{+}$, and form the interval $\left[d^{-}, d^{+}\right]$.

Theorem 2 was proved in the main text.

Proof of Theorem 3. Suppose that we have an unlimited number of processors (later on we will compute how many processors we actually need). First, let us compute $x_{i}^{+}=x_{i}+\varepsilon_{i}$ and $x_{i}^{-}=x_{i}-\varepsilon_{i}$ for all $i$. This can be done in parallel on $2 n$ processors. Since they are all working in parallel, we spend the time that is equivalent to only one computational step.

Now, we compute the values $\left(x_{i}^{+}-x_{j}^{-}\right)$and $\left(t_{i}-t_{j}\right)$ for $i>j$, and the values $\left(x_{j}^{-}-x_{i}^{+}\right)$ and $\left(t_{j}-t_{i}\right)$ for $j>i$. All these subtractions can also be done in parallel, and for that we 
need $2 n(n-1)$ processors (two for each pair $(i, j)$ except for $i=j$ ). The amount of time that we spend here is also equivalent to one computational step.

Next, we compute the ratios $\left(x_{i}^{+}-x_{j}^{-}\right) /\left(t_{i}-t_{j}\right)$ and $\left(x_{j}^{-}-x_{i}^{+}\right) /\left(t_{j}-t_{i}\right)$. This can also be done in parallel, on $n(n-1)$ processors, and takes the time of one computational step.

After that, we have two sets of $n(n-1) / 2$ numbers each; for the first set, we must find the biggest, for the second set, we must find the smallest. It is known (see, e.g., [8]), that for every $N$, the best method of finding the maximum of $N$ numbers $p_{1}, \ldots, p_{N}$ in parallel takes $\leq N$ processors, and can be done in $\leq \log _{2} N+1$ computational steps. For completeness, let us add a description of this algorithm:

$N=2$. If $N=2$, then we simply compare the two numbers $p_{1}$ and $p_{2}$

$N=4$. If $N=4$, then we divide the numbers into pairs $\left(p_{1}, p_{2}\right)$ and $\left(p_{3}, p_{4}\right)$. Then, first, we find the maximum for each pair: i.e., $\max \left(p_{1}, p_{2}\right)$ and $\max \left(p_{3}, p_{4}\right)$ (in parallel), and second, compare the two results to find the maximum of all four numbers $p_{i}$.

$N=8$. If $N=8$, then we divide the eight numbers into 4 pairs: $\left(p_{1}, p_{2}\right),\left(p_{3}, p_{4}\right),\left(p_{5}, p_{6}\right)$ and $\left(p_{7}, p_{8}\right)$. Then, for each pair, we compute its maximum: $q_{1}=\max \left(p_{1}, p_{2}\right), q_{2}=$ $\max \left(p_{3}, p_{4}\right), q_{3}=\max \left(p_{5}, p_{6}\right)$ and $q_{4}=\max \left(p_{7}, p_{8}\right)$. This can be done in parallel, and therefore takes the times of one computational step. After that, we find the maximum of the resulting 4 numbers $q_{1}, q_{2}, q_{3}, q_{4}$ (we already know that this can be done in 4 steps).

$N=2^{k}$. If $N=2^{k}$ for some $k$, then we have the following recursive procedure:

If $k=0$, then the only number present is the biggest;

If $k>0$, then we divide $N$ numbers into $2^{k-1}$ pairs, in one step find the biggest of all pairs, and then apply the same algorithm to find the biggest of $2^{k-1}$ results.

After each step, we halve the set of numbers that we still need to compare, so in $k$ steps we get the maximum.

$N \neq 2^{k}$. Let us now consider the remaining case, when $N \neq 2^{k}$. In this case, we take the smallest number $2^{k}$ that is $\geq N$, add $N-2^{k}$ numbers that are equal to $p_{1}$, and apply the same algorithm.

Since $k$ is the smallest for which $N \leq 2^{k}$, we have $N>2^{k-1}$, hence $\log _{2} N>k-1$, and $k<\log _{2}+1$. So, we need $\leq N$ processors and $\leq \log _{2} N+1$ computational steps. We can compute the minimum (to compute $d^{+}$) and the maximum (to compute $d^{-}$) in parallel. Each computation takes $\leq \log _{2}(n(n-1) / 2)+1<\log _{2}\left(n^{2} / 2\right)+1=2 \log _{2} n-1+1=2 \log _{2} n$ computational steps, and takes $\leq n(n-1) / 2$ processors. To compute both estimates in parallel, we need $\leq n(n-1)$ processors.

After we computed $d^{+}$and $d^{-}$, we must compare them to check whether $d^{+} \geq d^{-}$and thus whether $\mathcal{F}$ is compatible with $L$. This takes one more step.

Now we have described all the stages of our computation. In total, we need $\leq 1+1+$ $2 \log _{2} n+1=2 \log _{2} n+3$ computational steps, and $\leq \max (2 n, 2 n(n-1), n(n-1))=2 n(n-1)$ processors.

Proof of Theorem 4. In the algorithm from Theorem 2, the biggest part of computational time was spent on computing the expressions $\left(x_{i}^{-}-x_{j}^{+}\right) /\left(t_{i}-t_{j}\right)$ and $\left(x_{i}^{+}-x_{j}^{-}\right) /\left(t_{i}-t_{j}\right)$. In 
our case, we need these expressions for $n-b<j<i \leq n$. After we computed the estimates $d_{n}^{+}$and $d_{n}^{-}$, we read the new values $x_{n+1}$ and $t_{n+1}$. To compute $d_{n+1}^{+}$and $d_{n+1}^{-}$, we must use the similar expressions for $n+1-b<j<i \leq n+1$. But the values of these expressions for $i<n+1$ have already been computed on the previous stage, so we do not need to compute them again. The only new values that we have to compute correspond to $i=n+1$.

So, the new algorithm is as follows: for every $n$, we follow the same steps as in algorithm from Theorem 2, with the only difference that the values of $x_{i}^{-}, x_{j}^{+}$, and the expressions $\left(x_{i}^{-}-x_{j}^{+}\right) /\left(t_{i}-t_{j}\right)$ and $\left(x_{i}^{+}-x_{j}^{-}\right) /\left(t_{i}-t_{j}\right)$ that we have already computed on the previous stage (for $d_{n}^{+}$and $d_{n}^{-}$) we can use for $d_{n+1}^{+}$and $d_{n+1}^{-}$as well.

Let us estimate the number of computational steps per measurement for this algorithm. For a new value $t_{n+1}$, we must compute $(b-2)$ new pairs of expressions (one pair for each $j$ that lie between $n+1-b$ and $n+1$ ). To compute each pair, we need 5 operations, so totally, we need $5(b-2)$ computational steps.

We must add to this amount 2 computational steps to compute $x_{n+1}^{+}=x_{n+1}+\varepsilon_{n+1}$ and $x_{n+1}^{-}=x_{n+1}-\varepsilon_{n+1}$, and $2(b(b-1) / 2-1)$ steps to find the smallest and the biggest values $\left(d_{n+1}^{+}\right.$ and $\left.d_{n+1}^{-}\right)$. As a result, we get $2+5(b-2)+2(n(n-1) / 2-1)=2+5 b-10+b^{2}-b-2=b^{2}+4 b-10$.

Theorem 5 is proved in the main text.

Proof of Theorem 6. We consider the case when a function $x(t)$ is quadratic, i.e., when $x(t)=a+b\left(t-t_{0}\right)+c\left(t-t_{0}\right)^{2}$. In such a representation, the value of the derivative $d x(t) / d t$ in the point $t_{0}$ equals $b$.

If $n=2$, then for every $b$ and arbitrary values $x_{1}, x_{2}, \varepsilon_{1}>0$ and $\varepsilon_{2}>0$, we can easily find a function $x(t)$ that belongs to both $Q$ and $\mathcal{F}$ : indeed, it is sufficient to find $a$ and $c$ for which $a+b\left(t_{1}-t_{0}\right)+c\left(t_{1}-t_{0}\right)^{2}=x_{1}$ and $a+b\left(t_{2}-t_{0}\right)+c\left(t_{2}-t_{0}\right)^{2}=x_{2}$. These two equalities form a system of 2 linear equations with 2 unknowns $a$ and $b$ whose determinant is different from 0 , therefore this system always has a solution.

The same is true for $n=1$. So, if $n \leq 2$, then $\mathcal{F}$ is always consistent with $Q$, and any value of the derivative is possible, i.e., the derivative set is indeed equal to $(-\infty,+\infty)$.

Let us now consider the case $n>2$. A quadratic function $x(t)$ belongs to a function interval $\mathcal{F}$ if and only if $x_{i}^{-} \leq a+b\left(t_{i}-t_{0}\right)+c\left(t_{i}-t_{0}\right)^{2} \leq x_{i}^{+}$for all $i$ from 1 to $n$. We are interested in the value of $b$, so let us reformulate these inequalities in an equivalent form that does not contain $a$ and $c$. In other words, let us eliminate $a$ and $c$ from these inequalities.

First, let us eliminate $a$. This can be done in the same manner as in the proof of Theorem 1. Indeed, suppose that $b$ and $c$ are given. When does there exist an $a$ for which the above inequalities are true? If we move terms containing $b$ and $c$ to the other sides of the inequalities, we will obtain equivalent restrictions on $a$ :

$$
x_{i}^{-}-b\left(t_{i}-t_{0}\right)-c\left(t_{i}-t_{0}\right)^{2} \leq a \leq x_{i}^{+}-b\left(t_{i}-t_{0}\right)-c\left(t_{i}-t_{0}\right)^{2}
$$

So, $a$ must be not smaller than all the left-hand side expressions, and not bigger than all the right-hand side expressions. For such an $a$ to exist, every left-hand side expression must be not be greater than the right-hand side one, i.e., for every $i$ and $j$ the following inequality must be true:

$$
x_{i}^{-}-b\left(t_{i}-t_{0}\right)-c\left(t_{i}-t_{0}\right)^{2} \leq x_{j}^{+}-b\left(t_{j}-t_{0}\right)-c\left(t_{j}-t_{0}\right)^{2} .
$$

Since the new set of inequalities is equivalent to the old set, the problem of finding the derivative set can be now reformulated as follows: we must find all $b$ for which there exists a 
$c$ such that the inequalities

$$
x_{i}^{-}-b\left(t_{i}-t_{0}\right)-c\left(t_{i}-t_{0}\right)^{2} \leq x_{j}^{+}-b\left(t_{j}-t_{0}\right)-c\left(t_{j}-t_{0}\right)^{2}
$$

are true for all $i$ and $j$.

Let us now eliminate $c$ from this new set of inequalities. If we move all terms with $c$ into one side, and all other terms into another side, we get the following inequality:

$$
\left(x_{i}^{-}-x_{j}^{+}\right)-\left(b\left(t_{i}-t_{0}\right)-b\left(t_{j}-t_{0}\right)\right) \leq c\left(t_{i}-t_{0}\right)^{2}-c\left(t_{j}-t_{0}\right)^{2} .
$$

Since

$$
b\left(t_{i}-t_{0}\right)-b\left(t_{j}-t_{0}\right)=b\left(t_{i}-t_{0}-t_{j}+t_{0}\right)=b\left(t_{i}-t_{j}\right)
$$

and

$$
\begin{gathered}
c\left(t_{i}-t_{0}\right)^{2}-c\left(t_{j}-t_{0}\right)^{2}=c\left(\left(t_{i}-t_{0}\right)^{2}-\left(t_{j}-t_{0}\right)^{2}\right) \\
=c\left(\left(t_{i}-t_{0}\right)+\left(t_{j}-t_{0}\right)\right)\left(\left(t_{i}-t_{0}\right)-\left(t_{j}-t_{0}\right)\right)=c\left(t_{i}+t_{j}-2 t_{0}\right)\left(t_{i}-t_{j}\right)
\end{gathered}
$$

we can transform this inequality into the following simplified equivalent one:

$$
\left(x_{i}^{-}-x_{j}^{+}\right)-b\left(t_{i}-t_{j}\right) \leq c\left(t_{i}+t_{j}-2 t_{0}\right)\left(t_{i}-t_{j}\right) .
$$

For every pair $(i, j)$, we have this inequality, and we aiso have the inequality that is obtained from this one by changing $i$ and $j$.

The resulting inequality for $c$ depends on whether the coefficient at $c$ (equal to $\left.\left(t_{i}-t_{0}\right)^{2}-\left(t_{j}-t_{0}\right)^{2}\right)$ is negative, positive, or equal to 0 .

Let us first consider the case when $\left(t_{i}-t_{0}\right)^{2}=\left(t_{j}-t_{0}\right)^{2}$. Suppose for certainty that $i>j$. Then, we have two inequalities $\left(x_{i}^{-}-x_{j}^{+}\right)-b\left(t_{i}-t_{j}\right) \leq 0$, and $\left(x_{j}^{-}-x_{i}^{+}\right)-b\left(t_{j}-t_{i}\right) \leq 0$. Since $i>j$, we can reformulate these inequality as explicit restrictions on $b:\left(x_{i}^{-}-x_{j}^{+}\right) /\left(t_{i}-t_{j}\right) \leq$ $b \leq\left(x_{i}^{+}-x_{j}^{-}\right) /\left(t_{i}-t_{j}\right)$.

Now, let us consider the remaining case, when $\left(t_{i}-t_{0}\right)^{2} \neq\left(t_{j}-t_{0}\right)^{2}$. For certainty (and without losing generality), let us assume that $\left(t_{i}-t_{0}\right)^{2}>\left(t_{j}-t_{0}\right)^{2}$. Then the $i, j$ inequality is equivalent to the following:

$$
\frac{\left(x_{i}^{-}-x_{j}^{+}\right)-b\left(t_{i}-t_{j}\right)}{\left(t_{i}+t_{j}-2 t_{0}\right)\left(t_{i}-t_{j}\right)} \leq c
$$

and the $j, i$ inequality is equivalent to the following one:

$$
c \leq \frac{\left(x_{i}^{+}-x_{j}^{-}\right)-b\left(t_{i}-t_{j}\right)}{\left(t_{i}+t_{j}-2 t_{0}\right)\left(t_{i}-t_{j}\right)} .
$$

So, for a given $b$, the value of $c$ for which all the desired inequalities are true, exists if and only if first, all the inequalities that do not contain $c$ are true, and, second, any lower bound for $c$ is not greater than any upper bound for $c$, i.e.,

$$
\frac{\left(x_{i}^{-}-x_{j}^{+}\right)-b\left(t_{i}-t_{j}\right)}{\left(t_{i}+t_{j}-2 t_{0}\right)\left(t_{i}-t_{j}\right)} \leq \frac{\left(x_{k}^{+}-x_{l}^{-}\right)-b\left(t_{k}-t_{l}\right)}{\left(t_{k}+t_{l}-2 t_{0}\right)\left(t_{k}-t_{l}\right)}
$$

for each quadruple $(i, j, k, l)$ such that $\left(t_{i}-t_{0}\right)^{2}>\left(t_{j}-t_{0}\right)^{2}$ and $\left(t_{k}-t_{0}\right)^{2}>\left(t_{l}-t_{0}\right)^{2}$. 
We can express this inequality directly in terms of $b$ by moving all terms with $b$ into one side of the equation:

$$
\begin{gathered}
\frac{x_{i}^{-}-x_{j}^{+}}{\left(t_{i}+t_{j}-2 t_{0}\right)\left(t_{i}-t_{j}\right)}-\frac{x_{k}^{+}-x_{l}^{-}}{\left(t_{k}+t_{l}-2 t_{0}\right)\left(t_{k}-t_{l}\right)} \\
\leq \frac{b\left(t_{i}-t_{j}\right)}{\left(t_{i}+t_{j}-2 t_{0}\right)\left(t_{i}-t_{j}\right)}-\frac{b\left(t_{k}-t_{l}\right)}{\left(t_{k}+t_{l}-2 t_{0}\right)\left(t_{k}-t_{l}\right)} .
\end{gathered}
$$

Canceling similar terms in the numerator and the denominator of the fractions that are coefficients at $b$, we arrive at the following simplified inequality:

$$
\frac{x_{i}^{-}-x_{j}^{+}}{\left(t_{i}+t_{j}-2 t_{0}\right)\left(t_{i}-t_{j}\right)}-\frac{x_{k}^{+}-x_{l}^{-}}{\left(t_{k}+t_{l}-2 t_{0}\right)\left(t_{k}-t_{l}\right)} \leq b\left[\frac{1}{t_{i}+t_{j}-2 t_{0}}-\frac{1}{t_{k}+t_{l}-2 t_{0}}\right] \text {. }
$$

If $t_{i}+t_{j}-2 t_{0}=t_{k}+t_{l}-2 t_{0}$, then the coefficient at $b$ is equal to 0 , and therefore, this inequality turns into the following one:

$$
\frac{x_{i}^{-}-x_{j}^{+}}{\left(t_{i}+t_{j}-2 t_{0}\right)\left(t_{i}-t_{j}\right)}-\frac{x_{k}^{+}-x_{l}^{-}}{\left(t_{k}+t_{l}-2 t_{0}\right)\left(t_{k}-t_{l}\right)} \leq 0 .
$$

If $t_{i}+t_{j}-2 t_{0}=t_{k}+t_{l}-2 t_{0}>0$, then by multiplying both sides of the inequality by this number we conclude that $\left(x_{i}^{-}-x_{j}^{+}\right) /\left(t_{i}-t_{j}\right)-\left(x_{k}^{+}-x_{l}^{-}\right) /\left(t_{k}-t_{l}\right) \leq 0$, or $\left(x_{i}^{-}-x_{j}^{+}\right) /\left(t_{i}-\right.$ $\left.t_{j}\right) \leq\left(x_{k}^{+}-x_{l}^{-}\right) /\left(t_{k}-t_{l}\right)$. If $t_{i}+t_{j}-2 t_{0}=t_{k}+t_{l}-2 t_{0}<0$, then we get the inequality $\left(x_{i}^{-}-x_{j}^{+}\right) /\left(t_{i}-t_{j}\right) \geq\left(x_{k}^{+}-x_{l}^{-}\right) /\left(t_{k}-t_{l}\right)$.

Let us now consider the remaining case, when $t_{i}+t_{j}-2 t_{0} \neq t_{k}+t_{l}-2 t_{0}$. In this case, we actually have two inequalities: the one that we gave above, and the one that is obtained by changing $i, j$ to $k, l$ and vice versa. So, without losing generality, we can assume that $t_{i}+t_{j}-2 t_{0}>t_{k}+t_{l}-2 t_{0}$. In this case, $1 /\left(t_{i}+t_{j}-2 t_{0}\right)<1 /\left(t_{k}+t_{l}-2 t_{0}\right)$, the coefficient at $b$ is negative, and the inequality turns into the following:

$$
\left[\frac{x_{i}^{-}-x_{j}^{+}}{\left(t_{i}+t_{j}-2 t_{0}\right)\left(t_{i}-t_{j}\right)}-\frac{x_{k}^{+}-x_{l}^{-}}{\left(t_{k}+t_{l}-2 t_{0}\right)\left(t_{k}-t_{l}\right)}\right] /\left[\frac{1}{t_{i}+t_{j}-2 t_{0}}-\frac{1}{t_{k}+t_{l}-2 t_{0}}\right] \geq b .
$$

For the inequality that is obtained by changing $i, j$ to $k, l$, the coefficient at $b$ is positive, so we get the following inequality:

$$
\left[\frac{x_{i}^{+}-x_{j}^{-}}{\left(t_{i}+t_{j}-2 t_{0}\right)\left(t_{i}-t_{j}\right)}-\frac{x_{k}^{-}-x_{l}^{+}}{\left(t_{k}+t_{l}-2 t_{0}\right)\left(t_{k}-t_{l}\right)}\right] /\left[\frac{1}{t_{i}+t_{j}-2 t_{0}}-\frac{1}{t_{k}+t_{l}-2 t_{0}}\right] \leq b .
$$

We also have the bounds for $b$ from the cases when $\left(t_{i}-t_{0}\right)^{2}=\left(t_{j}-t_{0}\right)^{2}$ :

$$
\left(x_{i}^{-}-x_{j}^{+}\right) /\left(t_{i}-t_{j}\right) \leq b \leq\left(x_{i}^{+}-x_{j}^{-}\right) /\left(t_{i}-t_{j}\right) .
$$

So, we reduced the problem to the inequalities that do not depend on $b$ at all, and inequalities of the type $X \leq b$ and $b \leq Y$ for some expressions $X$ and $Y$. The inequalities that contain $b$ are consistent if and only if the maximum of the lower bounds $X$ is not bigger than the minimum of all upper bounds, and, if they are consistent, then the interval of possible values of $b$ coincides with the set of all possible values between these maximum and minimum. 
Proof of Theorem 7. The formulas from Theorem 6 prompt the following natural algorithm:

1) First, we compute the values $x_{i}^{+}=x_{i}+\varepsilon_{i}, x_{i}^{-}=x_{i}-\varepsilon, t_{i}-t_{0}$ and $\left(t_{i}-t_{0}\right)^{2}$ for all $i$ from 1 to $n$.

2) For every $i>j$, we check whether $\left(t_{i}-t_{0}\right)^{2}=\left(t_{j}-t_{0}\right)^{2}$.

2a) For all $i>j$, for which this equality is true, we compute the values $t_{i}-t_{j}, x_{i}^{+}-x_{j}^{-}$, $x_{i}^{-}-x_{j}^{+},\left(x_{i}^{+}-x_{j}^{-}\right) /\left(t_{i}-t_{j}\right)$, and $\left(x_{i}^{-}-x_{j}^{+}\right) /\left(t_{i}-t_{j}\right)$.

2b) If $\left(t_{i}-t_{0}\right)^{2} \neq\left(t_{j}-t_{0}\right)^{2}$, we permute $i$ and $j$ if necessary (so that $\left(t_{i}-t_{0}\right)^{2}>\left(t_{j}-t_{0}\right)^{2}$ ) and compute the values $t_{i}-t_{j},\left(t_{i}-t_{0}\right)+\left(t_{j}-t_{0}\right)=t_{i}+t_{j}-2 t_{0}, 1 /\left(t_{i}+t_{j}-2 t_{0}\right), x_{i}^{+}-x_{j}^{-}$, $x_{i}^{-}-x_{j}^{+},\left(\hat{x}_{i}^{+}-x_{j}^{-}\right) /\left(t_{i}-t_{j}\right),\left(x_{i}^{-}-x_{j}^{+}\right) /\left(t_{i}-t_{j}\right),\left(x_{i}^{+}-x_{j}^{-}\right) /\left(\left(t_{i}-t_{j}\right)\left(t_{i}+t_{j}-2 t_{0}\right)\right)$, and $\left(x_{i}^{-}-x_{j}^{+}\right) /\left(\left(t_{i}-t_{j}\right)\left(t_{i}+t_{j}-2 t_{0}\right)\right)$.

3) Enumerate all the pairs $i, j$ and $k, l$ such that $\left(t_{i}-t_{0}\right)^{2}>\left(t_{j}-t_{0}\right)^{2}$ and $\left(t_{k}-t_{0}\right)^{2}>\left(t_{l}-t_{0}\right)^{2}$. For each such quadruple, compare $t_{i}+t_{j}-2 t_{0}$ with $t_{k}+t_{l}-2 t_{0}$.

3a) If $t_{i}+t_{j}-2 t_{0}=t_{k}+t_{l}-2 t_{0}>0$, then check whether $\left(x_{k}^{+}-x_{l}^{-}\right) /\left(t_{i}-t_{j}\right) \geq\left(x_{i}^{-}-x_{j}^{+}\right) /\left(t_{i}-t_{j}\right)$. If this inequality is not true, then $\mathcal{F}$ is inconsistent with $Q$.

3b) If $t_{i}+t_{j}-2 t_{0}=t_{k}+t_{l}-2 t_{0}<0$, then check whether $\left(x_{j}^{+}-x_{i}^{-}\right) /\left(t_{j}-t_{i}\right) \geq\left(x_{l}^{-}-x_{k}^{+}\right) /\left(t_{l}-t_{k}\right)$. If this inequality is not true, then $\mathcal{F}$ is inconsistent with $Q$.

3c) If $t_{i}+t_{j}-2 t_{0}<t_{k}+t_{l}-2 t_{0}$, change $i, j$ to $k, l$ and vice versa, so that after that change we will have $t_{i}+t_{j}-2 t_{0}>t_{k}+t_{l}-2 t_{0}$.

3d) If $t_{i}+t_{j}-2 t_{0}>t_{k}+t_{l}-2 t_{0}$, then compute $N_{i j k l}^{+}, N_{i j k l}^{-}, D_{i j k l}, N_{i j k l}^{+} / D_{i j k l}$, and $N_{i j k l}^{-} / D_{i j k l}$ according to the formulas from the formulation of Theorem 6 .

4) After we have done the previous steps, we can compute $d_{1}^{-}=\max \left(\left(x_{i}^{-}-x_{j}^{+}\right) /\left(t_{i}-t_{j}\right)\right)$, $d_{1}^{+}=\min \left(\left(x_{i}^{+}-x_{j}^{-}\right) /\left(t_{i}-t_{j}\right)\right), d_{2}^{-}=\max \left(N_{i j k l}^{-} / D_{i j k l}\right), d_{2}^{+}=\min \left(N_{i j k l}^{+} / D_{i j k l}\right), d^{+}=$ $\min \left(d_{1}^{+}, d_{2}^{+}\right)$, and $d^{-}=\max \left(d_{1}^{-}, d_{2}^{-}\right)$. If $d^{+}<d^{-}$, then $\mathcal{F}$ is not compatible with $Q$, else $\left[d^{-}, d^{+}\right]$is the desired derivative set.

Let us now estimate the number of computational steps for this algorithm:

1) The first part of the algorithm requires 4 steps for every $i$ from 1 to $n$, i.e., overall, $4 n$ steps;

2) For every pair $i>j$, we make 1 comparison and then (depending on the result of this comparison) either 5 or 9 computational steps. Therefore, for each pair, we need at most 10 computational steps. Since there are $n(n-1) / 2$ pairs with $i>j$, we need at most $10 n(n-1) / 2=5 n(n-1)$ computational steps for this part of the algorithm.

3) For each quadruple $(i, j, k, l)$, we need 1 comparison, and then, depending on the result of this comparison, either 1 more comparison (in cases $3 \mathrm{a}$ and $3 \mathrm{~b}$ ), or 5 computational steps (in cases $3 \mathrm{c}$ and $3 \mathrm{~d}$ ). So, for every quadruple, we need at most 6 computational steps. 
To estimate the total number of steps for this part of the algorithm, we must multiply 6 by the total number of quadruples. A quadruple $(i, j, k, l)$ is a pair of different pairs $(i, j)$ and $(k, l)$. So, the total number of quadruples is equal to $P(P-1) / 2$, where $P$ is the total number of pairs with the property $\left(t_{i}-t_{0}\right)^{2}>\left(t_{j}-t^{0}\right)^{2}$. This number $P$ is not greater than the total number of pairs $n(n-1) / 2$, so the number of quadruples is $\leq P^{2} / 2 \leq(n(n-1) / 2)^{2} / 2$. Hence, the total number of compucational steps for this part of the algorithm is $\leq 6(n(n-1) / 2)^{2} / 2=3(n(n-1) / 2)^{2}$.

4) To compute the minimum $d_{2}^{+}$of $N \leq 1 / 2(n(n-1) / 2)^{2}$ numbers, we need $\leq N$ computational steps. Likewise, the number of steps for computing the maximum $d_{2}^{-}$ is $\leq 1 / 2(n(n-1) / 2)^{2}$. To compute the values $d_{1}^{+}$and $d_{1}^{-}$, we need $\leq n(n-1) / 2$ computational steps. Then, we need two steps to compute $d^{+}$and $d^{-}$. So, totally, we need $\leq(n(n-1) / 2)^{2}+n(n-1) / 2$ computational steps for this final part of the algorithm.

By adding all these numbers we can now get the estimate for the total amount of computational steps for the entire algorithm: it is $\leq S$, where $S=4 n+5 n(n-1)+3(n(n-$ $1) / 2)^{2}+(n(n-1) / 2)^{2}+n(n-1) / 2=4 n+11 n(n-1) / 2+4(n(n-1) / 2)^{2}$. If we compute all the expression in the parentheses, we arrive at the following result: $S=4 n+11 n^{2}-11 n+$ $n^{4}-2 n^{3}+n^{2}$. Adding all the coefficients at $1, n, n^{2}, n^{3}$, and $n^{4}$, we arrive at the following formula: $S=n^{4}-2 n^{3}+12 n^{2}-7 n<n^{4}+12 n^{2}$.

Proof of Theorem 8. We can compute all the values from parts 2), 3) of the previous proof in parallel: for that we need $\leq n^{4}$ processors (one for each quadruple). Totally, to compute all these expression, we need the time that is necessary for 6 computational steps:

1) On 1st step, we compute $x_{i}^{+}=x_{i}+\varepsilon_{i}, x_{i}^{-}=x_{i}-\varepsilon, t_{i}-t_{0}$, and $t_{i}-t_{j}$ for all $i$ and $j$.

2) On the 2nd step, we compute $\left(t_{i}-t_{0}\right)^{2},\left(t_{i}-t_{0}\right)+\left(t_{j}-t_{0}\right)=t_{i}+t_{j}-2 t_{0}, x_{i}^{+}-x_{j}^{-}$, and $x_{i}^{-}-x_{j}^{+}$for all $i$ and $j$.

3) On the 3rd step, for every $i>j$, we check whether $\left(t_{i}-t_{0}\right)^{2}=\left(t_{j}-t^{0}\right)^{2}$. For all $i>j$, for which this equality is true, we compute the values $\left(x_{i}^{+}-x_{j}^{-}\right) /\left(t_{i}-t_{j}\right)$ and $\left(x_{i}^{-}-x_{j}^{+}\right) /\left(t_{i}-t_{j}\right)$. If $\left(t_{i}-t_{0}\right)^{2} \neq\left(t_{j}-t^{0}\right)^{2}$, we permute $i$ and $j$ if necessary (so that $\left.\left(t_{i}-t_{0}\right)^{2}>\left(t_{j}-t^{0}\right)^{2}\right)$ and compute the values $1 /\left(t_{i}+t_{j}-2 t_{0}\right),\left(x_{i}^{+}-x_{j}^{-}\right) /\left(t_{i}-t_{j}\right)$, $\left(x_{i}^{-}-x_{j}^{+}\right) /\left(t_{i}-t_{j}\right)$.

4) On the 4 th step, we compute $\left(x_{i}^{+}-x_{j}^{-}\right) /\left(\left(t_{i}-t_{j}\right)\left(t_{i}+t_{j}-2 t_{0}\right)\right)$ and $\left(x_{i}^{-}-x_{j}^{+}\right) /\left(\left(t_{i}-\right.\right.$ $\left.\left.t_{j}\right)\left(t_{i}+t_{j}-2 t_{0}\right)\right)$ for all $i, j$.

5) On the joth step, we compute the values $N_{i j k l}^{ \pm}$and $D_{i j k l}$ for all $i, j, k, l$.

6) On the 6th step, we compute all the fractions $N_{i j k l}^{ \pm} / D_{i j k l}$.

Then, we must find the maximum and the minimum of $\leq(n(n-1))^{2}+n(n-1) / 2$ numbers. This amount is $\leq 1 / 4\left(n^{4}-2 n^{3}+n^{2}\right)+(1 / 2) n^{2}-(1 / 2) n=(1 / 4) n^{4}-(1 / 2) n^{3}+$ $(1 / 4) n^{2}+(1 / 2) n^{2}-(1 / 2) n=(1 / 4) n^{4}-(1 / 2) n^{3}+(3 / 4) n^{2}-(1 / 2) n$. Since $n \geq 3, n^{3} \geq 3 n^{2}$, so 
$(1 / 2) n^{3} \geq(3 / 2) n^{2}>(3 / 4) n^{2}$, so $(1 / 4) n^{4}-(1 / 2) n^{3}+(3 / 4) n^{2}-(1 / 2) n=(1 / 4) n^{4}-\left((1 / 2) n^{3}-\right.$ $\left.(3 / 4) n^{2}\right)-(1 / 2) n<(1 / 4) n^{4}$

Computing maximum and minimum can be done in parallel, so it is sufficient to estimate the time that is necessary to compute one of them, and then add 1 step for comparison of $d^{+}$and $d^{-}$. To find the smallest of $\leq(1 / 4) n^{4}$ numbers, we need $\leq \log _{2}\left((1 / 4) n^{4}\right)+1=$ $4 \log _{2} n-2+1=4 \log _{2} n-1$ steps.

Totally, we need $\leq 6+\left(4 \log _{2} n-1\right)=4 \log _{2} n+5$ computational steps.

Theorems 9 and 10 were proved in the main text.

Proof of Theorem 11. Let us first figure out what conditions on $a_{i}$ are equivalent to the demand that a statistical estimate is non-biased. This demand means that $E[d]=E\left[a_{1} x_{1}+\right.$ $\left.\cdots+a_{n} x_{n}\right]=\sum_{i} a_{i} E\left[x_{i}\right]=b$ for all $a, b$ and $x_{i}=a+b t_{i}+\xi_{i}$. Since $E\left[\xi_{i}\right]=0$, we conclude that $E\left[x_{i}\right]=a+b t_{i}$, hence $E[d]=\sum_{i} a_{i}\left(a+b t_{i}\right)=a\left(\sum_{i} a_{i}\right)+b\left(\sum_{i} t_{i}\right)$. So, the condition that $E[d]=b$ for all $a$ and $b$ means that $a\left(\sum_{i} a_{i}\right)+b\left(\sum_{i} t_{i}\right)=b$ for all $a$ and $b$. Two linear function are identical if and only if their coefficients coincide, therefore, this condition is true if $\sum_{i} a_{i}=0$ and $\sum_{i} a_{i} t_{i}=1$. So, these two equalities are necessary and sufficient for an estimate to be non-biased.

In the following we will consider only non-biased estimates. Let us compute the error for such estimates. Since $\xi_{i}$ are assumed to be independent, we can conclude that $\sigma^{2}[d]=$ $\sigma^{2}\left[\sum_{i} a_{i} x_{i}\right]=\sum_{i} \sigma^{2}\left[a_{i} x_{i}\right]$. But $x_{i}=a+b t_{i}+\xi_{i}$, therefore, $E\left[x_{i}\right]=a+b t_{i}, x_{i}-E\left[x_{i}\right]=\xi_{i}$, $\sigma^{2}\left[x_{i}\right]=E\left[\left(x_{i}-E\left[x_{i}\right]\right)^{2}\right]=E\left[\xi_{i}^{2}\right]=\sigma^{2}$, hence, $\sigma^{2}\left[a_{i} x_{i}\right]=a_{i}^{2} \sigma^{2}$ and $\sigma^{2}[d]=\sum_{i} \sigma^{2}\left[a_{i} x_{i}\right]=$ $\sigma^{2}\left(\sum_{i} a_{i}^{2}\right)$

So, the error is proportional to $\sqrt{\sum_{i} a_{i}^{2}}$. Hence, the error is the smallest possible if and only if the sum $\sum_{i} a_{i}^{2}$ is the smallest possible.

So, the problem of finding the non-biased statistical estimate with the smallest possible error, is equivalent to the following mathematical problem: to minimize a function $\sum_{i} a_{i}^{2}$ under the conditions $\sum_{i} a_{i}=0$ and $\sum_{i} a_{i} t_{i}=1$.

The Lagrange multipliers method allows us to reduce this problem to the unconditional optimization problem $F=\sum_{i} a_{i}^{2}+\lambda_{1} \sum_{i} a_{i}+\lambda_{2}\left(\sum_{i} a_{i} t_{i}-1\right) \rightarrow \min$ for some constants $\lambda_{i}$. This problem can be easily solved by equating the derivative $\partial F / \partial a_{i}$ of the minimized function $F$ to $0: 2 a_{i}+\lambda_{1}+\lambda_{2} t_{i}=0$. As a result, we get the expression $a_{i}=\alpha+\beta t_{i}$, where $\alpha=-1 / 2 \lambda_{1}$ and $\beta=-1 / 2 \lambda_{2}$. To find $\alpha$ and $\beta$, let us substitute these expressions into the conditions $\sum_{i} a_{i}=0$ and $\sum_{i} a_{i} t_{i}=1$. As a result, we get the following system of equations:

$$
\begin{aligned}
& \alpha \sum_{i} 1+\beta \sum_{i} t_{i}=0, \\
& \alpha \sum_{i} t_{i}+\beta \sum_{i} t_{i}^{2}=1 .
\end{aligned}
$$

From the first equation, we conclude that $\alpha=-\beta\left(\sum_{i} t_{i}\right) /\left(\sum_{i} 1\right)=-\beta\left(\sum_{i} t_{i}\right) / n=-\beta \bar{t}$. If we substitute this expression into the second equation, we conclude that

$$
\beta\left(\sum_{i} t_{i}^{2}-\left(\sum_{i} t_{i}\right) \bar{t} / n\right)=1
$$

The coefficient at $\beta$ can be easily represented as $\sum_{i}\left(t_{i}-\bar{t}\right)^{2}=n \sigma_{t}^{2}$, therefore, $\beta=1 /\left(n \sigma_{t}^{2}\right)$. Hence, $\alpha=-\bar{t} /\left(n \sigma_{t}^{2}\right)$, and $a_{i}=\alpha+\beta t_{i}=\left(t_{i}-\bar{t}\right) /\left(n \sigma_{t}^{2}\right)$. 
So, we have deduced the expressions for $a_{i}$ that correspond to the optimal statistical estimate. Let us now find the error of this estimate. This error is equal to $\sqrt{\sigma^{2} \sum_{i} a_{i}^{2}}=$ $\sigma \sqrt{\sum_{i} a_{i}^{2}}$. Substituing the above expression for $a_{i}$ into $\sum_{i} a_{i}^{2}$, we conclude that $\sum_{i} a_{i}^{2}=$ $\sum_{i}\left(\left(t_{i}-\bar{t}\right)^{2}\right)^{2} /\left(\sigma_{t}^{2} n\right)^{2}=\left(\sum_{i}\left(t_{i}-\bar{t}\right)^{2}\right)^{2} /\left(\sigma_{t}^{2} n\right)^{2}$. But $\left(\sum_{i}\left(t_{i}-\bar{t}\right)^{2}\right)^{2}=n \sigma_{t}^{2}$, therefore $\sum_{i} a_{i}^{2}=$ $1 /\left(\sigma_{t}^{2} n^{2}\right)$, and the error is equal to $\sigma \sqrt{\sum_{i} a_{i}^{2}}=\sigma /\left(\sigma_{t} n\right)$.

Proof of Theorem 12. If we know $a_{i}$, then after measuring $x_{i}$, we need only $2 n-1$ computational steps to compute the estimate $d=\sum_{i} a_{i} x_{i}: n$ multiplications and $n-1$ additions.

Proof of Theorem 13. If we have several computers working in parallel, then we can make all the muluplications in parallel (taking the time of only 1 computational step), and then compute the sum of the resulting $n$ products in the time of $\leq \log _{2} n+1$ computational steps (such an a!gorithm is given. e.g., in [8]) The idea of such an algorithm that parallelizes addition is very close to the idea of finding maximum in $\log _{2} n+1$ steps (see the proof of Theorem 3 ): if $n=2^{k}$ for some $k$, then we divide $n$ products $p_{1}=a_{1} x_{1}, p_{2}=a_{2} x_{2}, \ldots$ into pairs; on 1st step, we add each pair, getting the results $p_{1}+p_{2,} p_{3}+p_{4}, \ldots, p_{n-1}+p_{n}$; on 2 nd step, we divide these $n / 2$ results into pairs, and compute the sum of each pair (getting $p_{1}+p_{2}+p_{3}+p_{4}$, $\left.p_{5}+p_{6}+p_{6}+p_{7}+p_{8}, \ldots\right)$, etc.

Proof of Theorem 14. In this case, for a sequential algorithm, we need $n-1$ computational steps to compute the sum $\sum_{i} t_{i}, 1$ to compute $\bar{t}, 2 n$ to compute $t_{i}-\bar{t}$ and $\left(t_{i}-\bar{t}\right)^{2}, n-1$ to compute the sum $\sum_{i}\left(t_{i}-\bar{t}\right)^{2}, n$ to compute $a_{i}=\left(t_{i}-\bar{t}\right) /\left(\sum_{i}\left(t_{i}-\bar{t}\right)^{2}\right)$, and $2 n-1$ to compute $d=\sum_{i} a_{i} x_{i}$ : totally, we need $7 n-2$ computational steps.

Proof of Theorem 15. For a parallel computer, we need $\leq \log _{2} n+1$ steps to compute $\sum_{i} t_{i}$, 1 step to compute $\bar{t}, 2$ steps to compute $t_{i}-\bar{t}$ and $\left(t_{i}-\bar{t}\right)^{2}$ (because computations for different $i$ can be done in parallel), $\log _{2} n+1$ steps to compute the sum $\sum_{i}\left(t_{i}-\bar{t}\right)^{2}, 1$ step to compute $a_{i}=\left(t_{i}-\bar{t}\right) /\left(\sum_{i}\left(t_{i}-\bar{t}\right)^{2}\right)$ for all $i$, and $\log _{2} n+2$ steps to compute $d=\sum_{i} a_{i} x_{i}$. Totally, we need $3 \log _{2} n+8$ computational steps.

Proof of Theorem 16. Let us first reformulate the formula for $d$ from Theorem 11, so that is will become easier to compute in real time. According to Theorem 11, the optimal statistical estimate is equal to $d=\sum_{i} a_{i} x_{i}$, where $a_{i}=\left(t_{i}-\bar{t}\right) /\left(n \sigma_{t}^{2}\right)$ and $\sigma_{t}^{2}=\left(\sum_{i}\left(t_{i}-\bar{t}\right)^{2}\right) / n$. If we substitute the expression $\sigma_{t}^{2}=\left(\sum_{i}\left(t_{i}-\bar{t}\right)^{2}\right) / n$ into the formula for $a_{i}$, we conclude that $a_{i}=\left(t_{i}-\bar{t}\right) /\left(\sum_{i}\left(t_{i}-\bar{t}\right)^{2}\right)$. If we use the easily verifiable fact that $\sum_{i}\left(t_{i}-\bar{t}\right)^{2}=\sum_{i} t_{i}^{2} / n-n \bar{t}^{2}$, then we arrive at a formula $a_{i}=\left(t_{i}-\bar{t}\right) /\left(\sum_{i} t_{i}^{2}-n \bar{t}^{2}\right)$. Finally, if we substitute the expression for $\vec{t}$, we conclude that

$$
a_{i}=\left(t_{i}-\left(\sum_{i} t_{i}\right) / n\right) /\left(\sum_{i} t_{i}^{2}-n\left(\sum_{i} t_{i}\right)^{2}\right) .
$$

Therefore, $\left.d=\sum_{i} a_{i} x_{i}=\left(\sum_{i} x_{i} t_{i}-\left(\sum_{i} x_{i}\right) \dot{(} \sum_{i} t_{i}\right) / n\right) /\left(\sum_{i} t_{i}^{2}-\left(\sum_{i} t_{i}\right)^{2} / n\right)$. We can also express it in the following form: $d=\left(S_{1}-S_{2} S_{3} / n\right) /\left(S_{4}-S_{3}^{2} / n\right)$, where we denoted $S_{1}=$ $\sum_{i} x_{i} t_{i}, S_{2}=\sum_{i} x_{i}, S_{3}=\sum_{i} t_{i}$, and $S_{4}=\sum_{i} t_{i}^{2}$. In these terms, the error is equal to $e=\sigma / \sqrt{\sum_{i} t_{i}^{2}-n \bar{t}^{2}}=\sigma / \sqrt{S_{4}-S_{3}^{2} / n}$

For our case, a statistical estimate $d_{n}$ for a derivative in the moment $t_{n}$, is equal to $d_{n}=\left(S_{1, n}-S_{2, n} S_{3, n} / b\right) /\left(S_{4 n}-S_{3, n}^{2} / b\right)$, where 


$$
\begin{aligned}
& S_{1, n}=\sum_{i=n+1-b}^{n} x_{i} t_{i}, \quad S_{2 n}=\sum_{i=n+1-b}^{n} x_{i}, \\
& S_{3, n}=\sum_{i=n+1-b}^{n} t_{i},
\end{aligned}
$$

It is easy to check that $S_{1, n+1}=S_{1, n}+x_{n+1} t_{n+1}-x_{n+1-b} t_{n+1-b}, S_{2, n+1}=S_{2, n}+x_{n+1}-x_{n+1-b}$, $S_{3, n+1}=S_{3, n}+t_{n+1}-t_{n+1-b}$, and $S_{4, n+1}=S_{4, n}+t_{n+1}^{2}-t_{n+1-b}^{2}$. So, we can apply the following algorithm:

1) We reserve 4 variables $S_{1}, S_{2}, S_{3}, S_{4}$ that will contain the current values of the sums $S_{1, n}$. For every moment $t_{n}$, we also keep the values $x_{i}, x_{i} t_{i}$, $t_{i}$, and $t_{i}^{2}$ for all $i$ such that $n-b<i \leq n$. So, we must reserve $4+4 b$ variables.

2) The initial values of $S_{i, n}$ are 0 . When $n<b$ then after reading $x_{n}$ and $t_{n}$, we compute (and remember) the values $x_{n} t_{n}$ and $t_{n}^{2}$, and update the values $S_{i}$ in the following manner: $S_{1}:=S_{1}+x_{n} t_{n}, S_{2}:=S_{2}+x_{n}, S_{3}:=S_{3}+t_{n}$, and $S_{4}:=S_{4}+t_{n}^{2}$.

3) For $n=b$, we do this update, and also compute $d_{n}: \bar{t}:=S_{3} / b, S:=S_{4}-S_{3} \bar{t}$, $d_{n}:=\left(S_{1}-S_{2} \bar{t}\right) / S$, and $e:=\sigma / \sqrt{S}$.

4) For $n>b$, we must compute (and remember) the values $x_{n} t_{n}$ and $t_{n}^{2}$, and then update $S_{i}$ according to the following formulas: $S_{1}:=S_{1}+x_{n} t_{n}-x_{n-b} t_{n-b}, S_{2}:=S_{2}+x_{n}-x_{n-b}$, $S_{3}=S_{3}+t_{n}-t_{n-b}$, and $S_{4}:=S_{4}+t_{n}^{2}-t_{n-b}^{2}$. After that we compute $d_{n}$ and $e$ using the same formulas as for $n=b$.

For $n<b$, we need 6 computational steps; for $n=b$, we need $6+6=12$ steps to compute $d_{n}$, and one division + one square root to compute $e$. For $n>b$, we need 10 computational steps for an update, 6 to compute $d_{n}$, and 1 division +1 square root to compute $e$. In all cases, we need at most 17 arithmetic operations +1 square root per measurement.

Remurk. The algorithm described in this proof is vulnerable to errors: if accidentally we input a wrong value of $t_{i}$ for some $i$, then, we spoil $S_{1}, S_{3}, S_{4}$, and thus spoil the resulting estimates $d_{n}$ for arbitrary big $n$. To make this algorithm more error-prone, we can periodically check its results by applying the formulas from Theorem 11 directly. This checking will practically not slow down the computations, since we do not need to do it for every measurement, just periodically (e.g., for every tenth or every hundredth measurement).

Proof of Theorem 17. In this case, we can use an algorithm similar to the one from the proof of Theorem 16. Namely, if we have several processors that can work in parallel, then we can compute $x_{n} t_{n}$ and $t_{n}^{2}$ in parallel (thus, in the time that is necessary for 1 computational step), update the values $S_{i}$ in parallel on 4 processors (thus spending the time of 2 computational steps), and then compute the value $d_{n}$ as follows: first, compute $\bar{t}:=S_{3} / b$ (1 step), then $S:=S_{4}-S_{3} \bar{t}, s:=\sqrt{N}$, and $N:=S_{1}-S_{2} \bar{t}$ in parallel (2 steps), and $d_{n}:=N / S$ and $e:=\sigma / s$ (1 step). Totally, we need the time of $T$ computational steps.

Proof of Proposition 2. In this case, $\bar{t}=s_{0}$, and

$$
\sigma_{t}^{2}=1 /(2 k+1) \sum_{i}\left(t_{i}-\bar{t}\right)^{2}=(1 /(2 k+1)) \sum_{i=1}^{k}(i \Delta t)^{2}+\sum_{i=1}^{k}(i \Delta t)^{2}=\left(2 \Delta t^{2} /(2 k+1)\right) \sum_{i=1}^{k} i^{2}
$$

The value of the sum $\sum_{i=1}^{k} i^{2}$ is known to be $k(k+1)(2 k+1) / 6$ (this formula can be easily checked by mathematical induction). Therefore, $\sigma_{t}^{2}=2(1 /(2 k+1))(k(k+1)(2 k+1) / 6) \Delta t^{2}=$ 
$(k(k+1) / 3) \Delta t^{2}$. Substituting these expressions into the formulas from Theorem 11 completes the proof.

Proof of Proposition 3. In this case, $\vec{t}=s_{0}$, and

$$
\sigma_{t}^{2}=\frac{1}{n} \sum_{i}\left(t_{i}-\bar{t}\right)^{2}=\frac{\Delta t^{2}}{n} 2 \sum_{i=1 / 2}^{n-1 / 2} i^{2} .
$$

The values $i=1 / 2,3 / 2,5 / 2, \ldots,(n-1) / 2$ can be represented as $(2 j-1) / 2$ for $j=1,2, \ldots, k$. Here, $i^{2}=1 / 4(2 j-1)^{2}$, hence

$$
\sum_{i=1 / 2}^{n-1 / 2} i^{2}=1 / 4\left(\sum_{j=1}^{k}(2 j-1)^{2}\right) .
$$

The sum $S=\sum_{j=1}^{k}(2 j-1)^{2}$ of the square of all odd numbers from 1 to $n$ can be represented as the difference between the sum of all numbers from 1 to $n$ and all even numbers from 1 to $n$, i.e., as $S=\sum_{j=1}^{n} j^{2}-\sum_{j=1}^{n / 2}(2 j)^{2}$. The first sum is equal to $n(n+1)(2 n+1) / 6$, the second sum $\sum_{j=1}^{n / 2}(2 j)^{2}$ is equal to 4 times the sum $\sum_{j=1}^{n / 2} j^{2}$, i.e., to $4(n / 2)(n / 2+1)(2 n / 2+1) / 6=$ $n(n+2)(n+1) / 6$. Therefore, the difference between these two sums is equal to $n(n+1)(2 n+$ 1) $/ 6-n(n+2)(n+1) / 6=n(n+1)((2 n+1)-(n+2)) / 6=n(n+1)(n-1) / 6=n\left(n^{2}-1\right) / 6$. So, $\sigma_{t}^{2}=(2 / n)(1 / 4)\left(n\left(n^{2}-1\right) / 6\right)=\left(n^{2}-1\right) / 12$. Substituting these expressions into the formulas from Theorem 11 completes the proof.

Proof of Theorem 18. Here, $d=\left(\sum_{i} x_{i} t_{i}-\left(\sum_{i} x_{i}\right)(\bar{t})\right) /\left(n \sigma_{t}^{2}\right)$ The value $n \sigma_{t}^{2}$ is computed once (before the computations), so we can assume that it is already stored in the computer as some value $S$. So here, we have only three expressions to update: $S_{1}=\sum_{i} x_{i} t_{i}, S_{2}=\sum_{i} x_{i}$, and $\bar{t}$.

The value $\bar{t}$ is (as one can easily see) equal to the average of the first and the last values: $\bar{t}=1 / 2\left(t_{n}+t_{n+1-b}\right)=1 / 2\left(t_{1}+t_{1}+\Delta t n+\Delta t(n+1-b)\right)=t_{1}+\Delta t(n-(b-1) / 2)$, so its update can be done according to a simple formula: $\bar{t}:=\bar{t}+\Delta t$.

The update of $S_{1}$ and $S_{3}$ can be done by the same formulas as above, with the only difference that we cannot read $t_{n}$, we must compute it. This computation is also simple: $t_{n}:=t_{n-1}+\Delta t$

So, we arrive at the following algorithm: at every moment of time, we keep the values $S_{1}, S_{2} \bar{t}$, and the values $x_{i}, t_{i}$ and $x_{i} t_{i}$ for all $i$ such that $n-b<i \leq n$. Initially, $S_{1}$ and $S_{2}$ are set to $0, \bar{t}$ to $t_{1}-(b-1) / 2 \Delta t$, and $t_{1}$ to the given value.

Then, while $n<b$, we read $x_{n}$, compute $t_{n}:=t_{n-1}+\Delta t$ and $x_{n} t_{n}$, update $S_{1}$ and $S_{2}$. $S_{1}:=S_{1}+x_{n} t_{n}$ and $S_{2}:=S_{2}+x_{n}$, and update $\bar{t}:=\bar{t}+\Delta t$.

For $n=b$, in addition to all those steps, we compute the first estimate $d$ as $\left(S_{1}-S_{2} \bar{t}\right) / S$.

For $n>b$, we read $x_{n}$, compute $t_{n}:=t_{n-1}+\Delta t$ and $x_{n} t_{n}$, update $\bar{t}: \bar{t}:=\bar{t}+\Delta t$, update $S_{1}$ and $S_{2}$ using more complicated formulas: $S_{1}:=S_{1}+x_{n} t_{n}-x_{n-b} t_{n-b}, S_{2}:=S_{2}+x_{n}-x_{n-b}$, and compute $d$ as $\left(S_{1}-S_{2} \bar{t}\right) / S$.

For $n>b$, we need 10 computational steps; for $n \leq b$ we need less. So, our algorithm uses $\leq 10$ computational steps par measurement.

Proof of Theorem 19. Let us describe what can be parallelized in the algorithm from the previous proof: first, we can compute the new value of $t_{n}$, update $\bar{t}$ and start updating $S_{2}$ (1 step), then, on the 2 nd step, we end updating $S_{2}$, start updating $S_{1}$. On the 3 rd step, we 
the error is the smallest if and only if the sum $\sum_{i} a_{i}$ is the smallest possible. Applying Lagrange multipliers method to the resulting conditional optimization problem, we conclude that $a_{i}=\alpha+\beta\left(t_{i}-t_{0}\right)+\gamma\left(t_{i}-t_{0}\right)^{2}$. The linear equations for $\alpha, \beta$, and $\gamma$ are obtained, if we substitute the above expression for $a_{i}$ into the conditions $\sum_{i} a_{i}=0, \sum_{i} a_{i}\left(t_{i}-t_{0}\right)=1$, and $\sum_{i} a_{i}\left(t_{i}-t_{0}\right)^{2}=0$.

Proof of Theorem 21. According to Theorem 18, the optimal estimate $d$ is equal to $\alpha\left(\sum_{i} x_{i}\right)+$ $\beta\left(\sum_{i} x_{i}\left(t_{i}-t_{0}\right)\right)+\gamma\left(\sum_{i}\left(t_{i}-t_{0}\right)^{2}\right)$. So, if we already know how to compute $\alpha, \beta$, and $\gamma$, we will be able to compute $d$ in 5 steps ( 3 multiplications and 2 additions) if we update the three sums $\sum_{i} x_{i} ; \sum_{i} x_{i}\left(t_{i}-t_{0}\right)$, and $\sum_{i}\left(t_{i}-t_{0}\right)^{2}$ (just like sie did it in the proof of Theorem 16) The number of computational steps that is necessary to update a sum, does not depend on $b$.

To compute $\alpha, \beta$, and $\gamma$, we need to solve a system of 3 linear equations with 3 unknowns. If we know the coefficients, then the number of computational steps that we need to solve this system, does not depend on $b$.

All the coefficients are of the type $\sum_{i}\left(t_{i}-t_{0}\right)^{k}$ for $k=0,1,2,3,4$. The number of steps that we need to update these sums does not depend on $b$.

Adding all these numbers, we get the desired upper bound $C$ for the number of computational steps that does not depend on $b$.

Proof of Theorem 22. A linear estimate is uniquely determined by a sequence of coefficients $a_{1}, \ldots, a_{n}$. A precision $p$ of a linear estimate is defined as $p=\max \left|\sum_{i} a_{i} x_{i}-b\right|$ for all $a, b$ and $x_{i}$ such that $\left|x_{i}-\left(a+b t_{i}\right)\right| \leq \varepsilon$.

To simplify out proofs, let us introduce a new denotation: namely, let us denote $\Delta_{i}=$ $x_{i}-\left(a+b t_{i}\right)$. Then, $\left|\Delta_{i}\right| \leq \varepsilon, x_{i}=\left(a+b t_{i}\right)+\Delta_{i}$, and $d-b=\sum_{i} a_{i}\left(a+b t_{i}\right)+\sum_{i} a_{i} \Delta_{i}-b$. So, in these denotations, the definition of a precision $p$ can be reuritten as follows:

$$
p=\max _{a, b,\left|\Delta_{i}\right| \leq \varepsilon}\left|\sum_{i} a_{i}\left(a+b t_{i}\right)+\sum_{i} a_{i} \Delta_{i}-b\right|
$$

Let us first prove that if $\sum_{i} a_{i} \neq 0$, then $p=\infty$. Indeed, suppose that $\sum_{i} a_{i} \neq 0$. Let us fix ${ }^{i}$ some $b$ and $\Delta_{i}$ such that $\left|\Delta_{i}\right| \leq \varepsilon$, and consider the dependency of $s=\sum_{i} a_{i}\left(a+b t_{i}\right)+$ $\sum_{i} a_{i} \Delta_{i}-b$ on $a$. Since $s=\left(\sum_{i} a_{i}\right) a+\left(\sum_{i} a_{i} t_{i}-1\right) b+\sum_{i} a_{i} \Delta_{i}, s$ is a linear function of $a$ with a non-zero coefficient at 0 . Therefore, $|s| \rightarrow \infty$ as $a \rightarrow \infty$ and hence, $p=\max s=\infty$.

So, if $p<\infty$, then $\sum_{i} a_{i}=0$. Likewise, we can prove that if $p<\infty$, then $\sum a_{i} t_{i}=1$. Indeed, suppose that $\sum a_{i} t_{i} \neq 1$. Then, we can fix $a, \Delta_{i}$, and consider the dependency of $s$ on $b$. Here, $s$ is a linear function of $b$ with a non-zero coefficient, so $|s| \rightarrow \infty$ as $b \rightarrow \infty$ and hence, $p=\max s=\infty$.

So, if there is an estimate with finite precision $p$, then for this estimate, $\sum_{i} a_{i}=0$ and $\sum_{i} a_{i} t_{i}=1$. Let us consider any such estimate. For it $s=\sum_{i} a_{i} \Delta_{i}$. So,

$$
p=\max _{\left|\Delta_{i}\right| \leq \varepsilon}\left|\sum_{i} a_{i} \Delta_{i}\right| .
$$

Let us compute this maximum. Since $\Delta_{i}$ lies between $-\varepsilon$ and $\varepsilon$, the maximum possible value of $a_{i} \Delta_{i}$ is attained when $\Delta_{i}=\varepsilon$ if $a_{i}>0$, and when $\Delta_{i}=-\varepsilon$ if $a_{i}<0$. In both cases, this 
maximum value is equal to $\left|a_{i}\right| \varepsilon$. Therefore, the maximum possible value $p$ of the sum is equal to $\left(\sum_{i}\left|a_{i}\right|\right) \varepsilon$.

In particular, if we take $a_{i}$ from the formulation of the theorem (i.e., $a_{n}=-a_{1}=$ $1 /\left(t_{n}-t_{1}\right)$ and $\left.a_{2}=a_{3}=\cdots=a_{n-1}=0\right)$, we can easily check that $\sum_{i} a_{i}=0$ and $\sum_{i} a_{i} t_{i}=1$. So, for this estimate, the precision equals $\left(\sum_{i}\left|a_{i}\right|\right) \varepsilon=\left(2 /\left(t_{n}-t_{1}\right)\right) \varepsilon$. This value is finite, so, there always exists an estimate with finite precision.

Since $\varepsilon$ is fixed, the precision $p=\left(\sum_{i}\left|a_{i}\right|\right) \varepsilon$ is the smallest possible if and only if the sum $\sum_{i}\left|a_{i}\right|$ attains the smallest possible value. So, the problem of finding an estimate with the smallest possible precision is equivalent to the problem of finding a sequence of real numbers $a_{1}, \ldots, a_{n}$ that satisfies the following conditional optimization problem: $\sum_{i}\left|a_{i}\right| \rightarrow$ min under the condition that $\sum_{i} a_{i}=0$ and $\sum_{i} a_{i} t_{i}=1$.

Let us first prove that this problem has a solution. Indeed, we already know a sequence $a_{i}$ that satisfies both conditions. For this sequence, $\sum_{i}\left|a_{i}\right|=2 /\left(t_{n}-t_{1}\right)$. Therefore, while looking for the most precise estimate, we can restrict ourselves by the sequences for which $\sum_{i}\left|a_{i}\right| \leq 2 /\left(t_{n}-t_{1}\right)$. For such sequences, for every $i,\left|a_{i}\right| \leq \sum_{i}\left|a_{i}\right| \leq 2 /\left(t_{n}-t_{1}\right)$. The set of the sequences for which $\sum_{i}\left|a_{i}\right| \leq 2 /\left(t_{n}-t_{1}\right)$ is bounded, and it is also closed. Hence, it is compact. The conditions $\sum_{i} a_{i}=0$ and $\sum_{i} a_{i} t_{i}=1$ also determine closed sets. Therefore, the set $S$ of all sequences, for which $\sum_{i}\left|a_{i}\right| \leq 2 /\left(t_{n}-t_{1}\right), \sum_{i} a_{i}=0$ and $\sum_{i} a_{i} t_{i}=1$, is an intersection of a compact set and a closed set, and is therefore a compact. A function $\sum_{i}\left|a_{i}\right|$ is continuous on this compact set $S$, therefore, it attains its minimum in some point. So, there exists a sequence, for which the sum $\sum_{i}\left|a_{i}\right|$ takes the smallest possible value. As we have already mentioned, this means that this sequence has the smallest possible value of $p$.

Let us now find the sequence with the smallest possible value of $p$ (or, what is equivalent, the smallest possible value of $\left.\sum_{i}\left|a_{i}\right|\right)$. Let $a_{i}$ be such sequence. Let us prove that at most two of its elements are different from 0 . We will prove it by showing that if three different elements of $a_{i}$ are different from 0 , then this sequence cannot be the one for which $\sum_{i}\left|a_{i}\right|$ is the smallest possible. Indeed, suppose that $a_{i} \neq 0$ for at least 3 different indices $i$. Since $\sum_{i} a_{i}=0$, the values of $a_{i}$ cannot all be of one sign. So, we can choose the three of them that are not all of one sign (i.e., either one is positive and two other are negative, or one is negative, and two other are positive). Let us denote these three values by $j$, and $l$. So, $a_{j} \neq 0$, $a_{k} \neq 0$, and $a_{l} \neq 0$ for some $j<k<l$.

Let us prove that by changing these three values we can diminish the value of $\sum_{i}\left|a_{i}\right|$, while still retaining the conditions $\sum_{i} a_{i}=0$ and $\sum_{i} a_{i} t_{i}=1$. We will try to take a sequence $a_{i}^{\prime}$ that is defined by the formulas $a_{j}^{\prime}=a_{j}+\alpha b_{j}, a_{k}^{\prime}=a_{k}+\alpha b_{k}$, and $a_{l}^{\prime}=a_{l}+\alpha b_{l}$ for some real numbers $\alpha \neq 0, b_{j}, b_{k}, b_{l}$, and $a_{i}^{\prime}=a_{i}$ for all $i$ that are different from $j, k$, or $l$.

We want to guarantee that $\sum_{i} a_{i}^{\prime}=0$ and $\sum_{i} a_{i}^{\prime} t_{i}=1$. We assumed that the sequence $a_{i}$ satisfies these conditions. So, if we substitute the above expressions for $a_{i}^{\prime}$ into these formulas, and use the conditions that $\sum_{i} a_{i}=0$ and $\sum_{i} a_{i} t_{i}=1$, we can conclude that $a_{i}^{\prime}$ satisfies these conditions if and only if $b_{j}+b_{k}+b_{l}=0$ and $b_{j} t_{j}+b_{k} t_{k}+b_{l} t_{l}=0$. The first equation is satisfied if $b_{l}=-\left(b_{j}+b_{k}\right)$. Substituting this value into the second equation, we obtain the equation $b_{j}\left(t_{j}-t_{l}\right)+b_{k}\left(t_{k}-t_{l}\right)=0$. Therefore, the second condition is satisfied, if we take an arbitrary $b_{j}$, and $b_{k}=-b_{j}\left(t_{l}-t_{j}\right) /\left(t_{l}-t_{k}\right)$. From these two expression, we conclude that $b_{l}=-\left(b_{j}+b_{k}\right)=b_{j}\left(t_{k}-t_{j}\right) /\left(t_{l}-t_{k}\right)$.

So, if we take an arbitrary $b_{j}, b_{k}=-b_{j}\left(t_{l}-t_{j}\right) /\left(t_{l}-t_{k}\right)$, and $b_{l}=b_{j}\left(t_{k}-t_{j}\right) /\left(t_{l}-t_{k}\right)$, then the resulting values $a_{i}^{\prime}$ satisfy both conditions $\sum_{i} a_{i}^{\prime}=0$ and $\sum_{i} a_{i}^{\prime} t_{i}=1$.

Let us now estimate $\sum_{i}\left|a_{i}^{\prime}\right|$ for these $a_{i}^{\prime}$. Since $a_{j}+\alpha b_{j} \rightarrow a_{j}$ as $\alpha \rightarrow 0$, we can conclude 
that for sufficiently small $\alpha$, the expression $a_{j}+\alpha b_{j}$ has the same sign as $a_{j}$. Therefore, for such $\alpha,\left|a_{j}+\alpha b_{j}\right|=\operatorname{sign}\left(a_{j}+\alpha b_{j}\right)\left(a_{j}+\alpha b_{j}\right)=\operatorname{sign}\left(a_{j}\right)\left(a_{j}+\alpha b_{j}\right)=\left|a_{j}\right|+\operatorname{sign}\left(a_{j}\right) \alpha b_{j}$. Similar expressions are true for $k$ and $l$, therefore, for sufficiently small $\alpha, \sum_{i}\left|a_{i}^{\prime}\right|=\sum_{i}\left|a_{i}\right|+\alpha \Sigma$, where $\Sigma=\operatorname{sign}\left(a_{j}\right) b_{j}+\operatorname{sign}\left(a_{k}\right) b_{k}+\operatorname{sign}\left(a_{l}\right) b_{l}$. Let us prove that $\Sigma \neq 0$. Indeed, we chose $j, k$ and $l$ in such a way that two of the values $a_{j}, a_{k}, a_{l}$ are of one sign, and the third value is of different sign. Suppose, for example that $a_{j}>0, a_{k}>0$ and $a_{l}<0$. Then, $\Sigma=b_{j}+b_{k}-b_{l}$. We have already proved that $b_{j}+b_{k}+b_{l}=0$, therefore, $\Sigma=\left(b_{j}+b_{k}+b_{l}\right)-2 b_{l}=-2 b_{l}$. According to the above expression for $b_{l}$, it is $\neq 0$ if $b_{j} \neq 0$. So, in this cases, $\Sigma \neq 0$. Likewise, we can consider all other combinations of signs and prove that in all cases, $\Sigma \neq 0$.

Since $\Sigma \neq 0$, we can take $\alpha$ of the opposite sign with $\Sigma$, and find a sequence $a_{i}^{\prime}$, for which $\sum_{i}\left|a_{i}^{\prime}\right|<\sum_{i}\left|a_{i}\right|$, and thus, a contradiction to our assumption that $\sum_{i}\left|a_{i}\right|$ attained its minimum in the given sequence $a_{i}$. This contradiction proves that the assumption that $a_{i}$ is non-zero for at least 3 different $i$, is false.

So, $a_{i}$ is different from 0 for at most 2 different values of $i$. It cannot be different from 0 only for one $i$, because from $\sum_{i} a_{i}=0$ we would then conclude that $a_{i} \equiv 0$, and hence $\sum_{i} a_{i} t_{i}=0 \neq 1$. Therefore, for optimal sequence $a_{i}, a_{i}$ is different from 0 precisely for 2 different values $j<k$.

In this case, the conditions $\sum_{i} a_{i}=0$ and $\sum_{i} a_{i} t_{i}=1$ turn into $a_{j}+a_{k}=0$ (hence $\left.a_{j}=-a_{k}\right)$ and $a_{j} t_{j}+a_{k} t_{k}=1$. Substituting $a_{j}=-a_{k}$ into the second equation, we conclude that $a_{k}=1 /\left(t_{k}-t_{j}\right)$ and $a_{j}=-1 /\left(t_{k}-t_{j}\right)$. For this sequence, $\sum_{i}\left|a_{i}\right|=2 /\left(t_{k}-t_{j}\right)$.

So, in order to find a sequence $a_{i}$ with the smallest possible precision $p$, we must find a pair $j<k$, for which the expression $2 /\left(t_{k}-t_{j}\right)$ takes the smallest possible value. This expression is the smallest possible if and only if the difference $t_{k}-t_{j}$ is the biggest possible. This is attained if $t_{k}$ is the biggest possible (i.e., $k=n$ ), and $t_{j}$ is the smallest possible (i.e., $j=1$ ). Therefore, the sequence $a_{i}$ for which $p$ is the smallest coincides with the one given in the formulation of the theorem.

\section{Acknowledgements}

This work was supported by a NSF Grant No. CDA-9015006, NASA Research Grant No. 9482, and a grant from GSA, administered by Materials Research Institute and the Institute for Manufacturing and Materials Management. The authors are thankful to Misha Koshelev who tested our sequential algorithms by programming them in Pascal, to Sergey Shary and Vyacheslav Nesterov for valuable discussions and important preprints, and to the anonymous referees for their thoroughness and help.

\section{References}

[1] Aberth, O. Precise numerical analysis. Wm. C. Brown Publ., Dubuque, Iowa, 1988.

[2] Bellman, R. Introduction to matrix analysis. McGraw Hill, N.Y., 1970.

[3] Bohlender, G., Ulrich, C., Wolff von Gudenberg, J., and Rall, L. B. Pascal-SC. A compuler langruage for scientific comprututions. Academic Press, N.Y., 1987. 
APPLICATIONS OF INTERVAL COMPUTATIONS TO EARTHQUAKE-RESISTANT ENGINEERING...

[4] Burden, R. L. and Faires, J. D. Numerical analysis. Prindle, Weber \& Schmidt, Boston, MA, 1985.

[5] Chung, L. L., Lin, R. C., Soong, T. T., and Reinhorn, A. M. Experimental study of active control for MDOF seismic structures. Journal of Engineering Mechanics 115 (8) (1989), pp. 1609-1627.

[6] Corliss, G. F. Applications of differentiation arithmetic. In: Moore, R. E. (ed.) "Reliability in computing", Academic Press, N.Y., 1988, pp. 127-148.

[7] Dehghanyar, T. J., Masri, S. F., Miller, R. K., and Caughey, T. K. On-line purameter control of nonlinear flexible structures. In: "Proceedings of the 2nd International Symposium on Structural Control, Martinus-Nijhoff, Boston, MA, 1987”, pp. 141-159.

[8] Jaja, J. An introduction to parallel algorithns. Addison-Wesiey, Reading, MA, 1992.

[9] Karmarkar, N. A new polynomial-time algorithm for linear programming. Combinatorica 4 (1984), pp. $373-396$.

[10] Kobori, T. Siate-of the-art of structural comtrol resentch in Japan. In: "Proceedings of the US National Workshop on Structural Control, October 25-26, 1990", pp. p1-p21.

[11] Kobori, T. et al. U.S. Putent No. 5,036,633.

[12] Kobori, T., Kanayama, H., and Kamagata, S. A proposal of new anli-seismic structure wilh active seismic response conlrol system - dymamic intelligent building. In: "Proceedings of the 9 th World Conference on Earthquake Engineering, Tokyo-Kyoto, Japan, VIIn", pp. 465-470.

[13] Kobori, T., Kanayama, H., and Kamagata, S. Active seismic response conlrol systems for nuclear power plant equipment facilities. Nuclear Engineering and Design 111 (1989), pp. 351-356.

[14] Kobori, T., Yamada, S., and Kamagata, S. U.S. Putent No. 4,922,667.

[15] Kreinovich, V., Lakeyev, A., and Noskov, S. Optimal soluuion of inlerval linear systerns is intractable (NP-hard). Interval Computations (1) (1993).

[16] Markov, S. Interval differential equations. In: Nickel, K. E. (ed.) "Interval Mathematics 1980", Academic Press, N.Y., 1980, pp. 145-164.

[17] Meirovitch, L., Baruch, H., and Oz, H. A comparison of control lechniques for large flexible structures. Journal of Guidance 6 (4) (1983), pp. 302-310.

[18] Moore, R. E. Automatic local coordinale iransformations to reduce the growth of error bonnds in interval computaion of solution of ordinary differential equations. In: Rall, L. B. (ed.) "Errors in Digital Computations, Proceedings of a Symposium", John Wiley \& Sons, N.Y., 1965, pp. 103-140.

[19] Moore, R. E. Interval analysis. Prentice Hall, Englewood Cliffs, N.J., 1966.

[20] Moore, R. E. Mathematical elements of scientific compruing. Holt, Rinehart and Winston, N.Y., 1975.

[21] Moore, R. E. Methods and applications of interval analysis. SIAM, Philadelphia, 1979. 
[22] Nemir, D. C., Koivo, A. J., and Kashyap, R. L. Psendolinks and the self-tuning comtrol of a non-rigid link mechanism. IEEE Transactions on Systems, Man and Cybernetics 18 (1) (1988), pp. $40-48$.

[23] Osegueda, R. A., Nemir, D. C., and Lin, Y. J. On-line adaptive stiffness control to tailor modal energy contents in structures. In: "ADPA/ALAA/ASME/SPIE Conference on Active Materials and Adaptive Structures, Alexandria, VA, November 5-8, 1991".

[2.4] Rall, L. B. Applications of softuare for antomatic differentiution in numerical compulation. In: Alefeld, G. and Grigorieff, R. D. (eds) "Fundamentals of Numerical Computations (ComputerOriented Numerical Analysis)", Springer-Verlag, Wien, N.Y., 1980, pp. 141-15̄6.

[25] Rall, L. B. Automutic differentiution: techiques and upplications. Lecture Notes in Computer Science 120, Springer-Verlag, Berlin-Heidelberg-N.Y., 1981.

[26] Rall, L. B. Differentiation and generation of Taylor coefficients in Pascal-SC. In: Kulisch, U. W. and Miranker, W. L. (eds) "A New Approach to Scientific Computation". Academic Press, N.Y., 1983, pp. 291-309.

[2i] Ratschek, H. and Schröder, G. Über die Abteilung zom intervallwertigen Funktionen. Computing 7 (19i1), pp. 172-187.

[28] Sendov, B. Some topics of segment analysis. In: Nickel, K. E. (ed.) "Interval Mathematics 1980", Academic Press, N.Y., 1980, pp. 203-222.

[29] Shary, S. P. Solution of "outer" and "inner" problems for an interwal system of linear algebraic equatioms. Ph.D. Dissertation, Kransoyarsk-Ekaterinburg, 1991 (in Russian).

[30] Shary, S. P. A new class of algarithms for optimal solution of interval linear systems. Interval Computations (2) (1992), pp. 18-29.

[31] Soong, T. T. Active structural control: theory and practice. Longman Scientific, Essex, 1990.

Received: May 25, 1992

Revised version: January 12, 1994
Electrical Engineering Department and Computer Science Department University of Texas at El Paso

El Paso, TX 79968 